\title{
Tracking the Tracker from its Passive Sonar ML-PDA Estimates
}

\author{
Domenico Ciuonzo*, Student Member, IEEE, Peter K. Willett $\dagger$, Fellow, IEEE, \\ Yaakov Bar-Shalom $\dagger$, Fellow, IEEE
}

* Dept. of Industrial and Information Engineering, Second University of Naples, Aversa, (CE), Italy.

$\nmid$ †ept. of Electrical and Computer Engineering, University of Connecticut, Storrs, (CT), USA.

Email: domenico.ciuonzodunina2.it,

$\{$ willett, ybs\} @engr.uconn.edu.

\begin{abstract}
Target motion analysis with wideband passive sonar has received much attention. Maximumlikelihood probabilistic data-association (ML-PDA) represents an asymptotically efficient estimator for deterministic target motion, and is especially well-suited for low-observable targets; the results presented here apply to situations with higher signal to noise ratio as well, including of course the situation of a deterministic target observed via "clean" measurements without false alarms or missed detections. Here we study the inverse problem, namely, how to identify the observing platform (following a "two-leg" motion model) from the results of the target estimation process, i.e. the estimated target state and the Fisher information matrix, quantities we assume an eavesdropper might intercept. We tackle the problem and we present observability properties, with supporting simulation results.
\end{abstract}

\section{Index Terms}

Eavesdropper, Fisher information matrix, ML-PDA, nonlinear identification, platform localization, stealthy platform, wideband passive sonar.

P. K. Willett was supported by ONR under contracts N00014-09-10613 and N10014-10-10412. Y. Bar-Shalom was supported by ARO under contract W911NF-10-10369 and ONR under contract N00014-10-1-0029. 


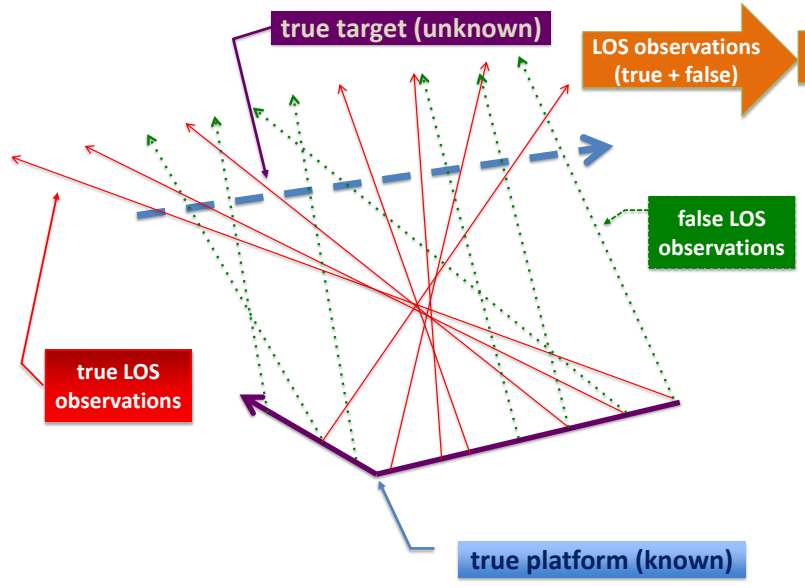

(a) Collecting measurements.

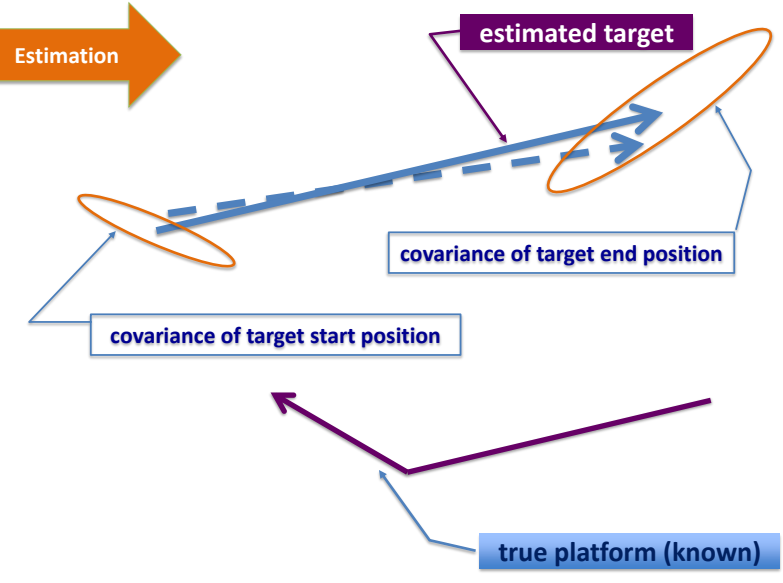

(b) Estimating target trajectory.

Figure 1: The target estimation process by the platform side.

\section{INTRODUCTION}

\section{A. Problem Motivation}

Target motion analysis (TMA) with bearings-only measurements is a well-understood and extensively studied problem (see Fig. 1). It has been shown in the literature that as long as the platform is outmaneuvering the target, observability of the latter is assured and its motion can be inferred, even from very noisy measurements. Conversely, it is useful to understand whether, given the results of TMA estimation, it could be possible to identify, completely or at least partially, the trajectory of the observing platform.

More specifically, the problem arises when a "target-friendly" entity, as opposed to being cooperative with the platform, intercepts the results of the target estimation performed by the platform (see Fig. 2); the question is whether this entity can identify, partially or totally, the trajectory of the platform. The feasibility of this problem is of twofold interest: $(i)$ it verifies the utility of intercepting communications (containing TMA-related information) between the platform and platform-cooperative entities, because this information would be useful; and (ii) it motivates, at the platform side, the need for secure and encrypted transmission of the TMA estimation results. 


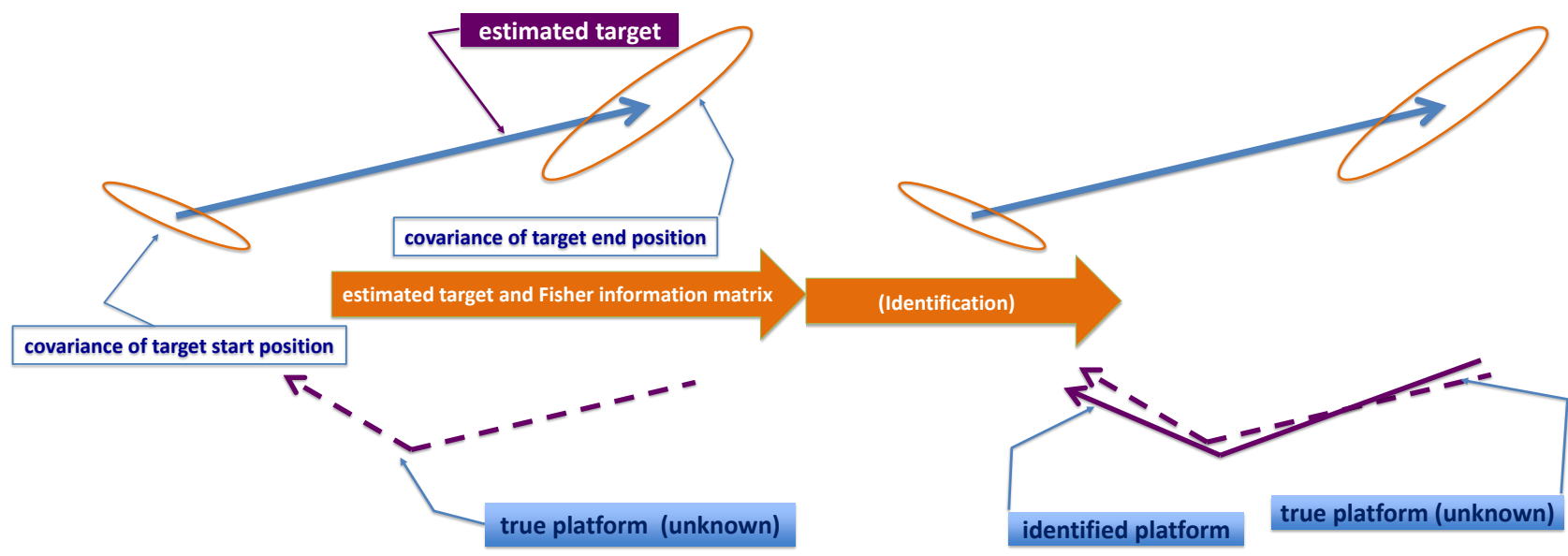

(a) Intercepting target estimation results from the platform side.

(b) Identifying the platform trajectory.

Figure 2: The platform identification process by the target-friendly side.

\section{B. Related Works}

Seminal results on the continuous-time observability of the target motion, through a wideband passive sonar, were derived in [23], [13]. In fact, by successive differentiations of the measurement function, a necessary condition was derived and it was shown that a platform maneuver is a needed prerequisite to ensure observability of the target; however unobservable maneuveringplatform trajectories could exist (i.e. the platform may still take a trajectory wherefrom the target is unobservable). This analysis was rigorously extended, in the form of a necessary and sufficient condition, in [26], [12], [4], [18]; a comprehensive analysis of observability related to practical scenarios was also conducted in [18]. These results were also demonstrated in discretetime in [14] via linear algebra; observability insights in different scenarios were presented, and also a stochastic observability (and estimability) analysis was performed. In [16] a theoretical connection between the invertibility of the Fisher information matrix (FIM) and target (local) observability was established. In [25] (and references therein) the optimal platform maneuver was designed, in the sense of the best estimation accuracy in terms of the FIM.

There are three common approaches to standard TMA (with or without Doppler measurements): maximum likelihood (ML), pseudolinear (PL) and instrumental variables (IV) estimation. Although the first approach is asymptotically efficient, it is complex and therefore suboptimal 
solutions are desirable. PL estimation has the advantage of being in closed form and of easy computation; however it can lead to severe bias even in favorable conditions [9]. Consequently IV estimation, yielding estimates with reduced bias, has seen recent attention [15], [10], [9], [11], [28].

Alternative bearings-only TMA scenarios have been studied recently in [3], [19], [8] and ML batch estimators have been proposed. More specifically, in [3] the problem of bearingsonly TMA for conditionally-deterministic target motion and with operational constraints on the platform is tackled with Markov chain Monte Carlo methods. In [19] TMA of a maneuvering target and non-maneuvering platform is studied; observability is established and a batch estimator is proposed. The concept was later applied to the scenario of a circular constant-speed target and a non-maneuvering target in [8].

The estimators proposed in these references do not deal with the problem of false measurements (clutter) and less-than-unity probability of detection. The seminal work in [17] derived a ML estimate of target parameters for both wideband and narrowband passive sonars in the presence of false detections (clutter), based on probabilistic data association (ML-PDA); the performance of the estimator was evaluated in terms of the Cramér-Rao lower bound (CRLB). It was shown that the effect of the clutter on the performance through the CRLB was simply via a product with a less-than-unity scalar value, called the information reduction factor (IRF).

The ML-PDA was extended by incorporating amplitude information to enhance performance in the scenarios of "low-observable" (i.e. low Signal-to-Noise ratio) targets in [21]; improved accuracy and superior global convergence were demonstrated. In [7] ML-PDA was applied to the problem of low-observable target estimation using electro-optical sensors; also a slidingwindow batch approach for ML-PDA estimation was derived, capable of dealing with temporary disappearance of targets and/or targets with velocities changing over time. ML-PDA was also successfully applied to active sonar tracking in [6], where also an efficient computation of the ML estimate, namely, directed subspace search (DSS), was derived. The use of ML-PDA for early track detection with a radar is discussed in [2].

\section{Main Results and Paper Organization}

The main contributions of the present paper are summarized as follows: 
- We study the "inverse" problem of identifying the platform trajectory, following a "twoleg" motion model, through its ML-PDA estimation results on a target; to the best of our knowledge, such a problem is addressed here for the first time.

- We derive and study the objective function to be optimized for identifying the platform trajectory; it is shown that the optimization of this function depends on neither the IRF nor the measurement variance at the platform side; that is, the exact ${ }^{1}$ information to identify the platform trajectory is unnecessary.

- Also it is demonstrated that the platform trajectory is unobservable unless it keeps a constant speed during its two different legs.

- We use an efficient and practical algorithm, based on a derivative-free local search, to solve the nonlinear problem associated with the identification task. The local optimization routine is initialized from geometric considerations and exploits the structure of the observed FIM. The paper is organized as follows: In Sec. II we introduce the model for passive wideband-sonar localization and we give the background on the ML-PDA approach; in Sec. III we formulate the problem of inverse localization and we show some important identification properties; in Sec. IV we devise a procedure to compute a good initial estimate as input for the local optimization routines, while in Sec. V we show, by simulation, the performance of the proposed solution; some concluding remarks and future research are given in Sec. VI; proofs and derivations are confined to the Appendices.

Notation - Lower-case (resp. Upper-case) bold letters denote vectors (resp. matrices), with $a_{n}$ (resp. $\left.A_{n, m}\right)$ representing the $n$th (resp. the $(n, m)$ th) element of the vector $\boldsymbol{a}$ (resp. matrix $\boldsymbol{A}$ ); upper-case calligraphic letters and braces denote finite sets, with $[a: b], a \leq b$, representing the set $\{a, a+1, \ldots, b\} ; \boldsymbol{I}_{N}$ denotes the $N \times N$ identity matrix, while $\operatorname{diag}(\boldsymbol{t})$ is a diagonal matrix with diagonal equal to $t ;(\cdot)^{t},\|\cdot\|_{2},\|\cdot\|_{F}$ and $\langle\cdot\rangle$ denote transpose, $\ell_{2}$ norm, Frobenius norm and inner product operators, respectively; $\nabla_{\boldsymbol{t}}(\cdot)$ denotes the gradient operator w.r.t. the vector $\boldsymbol{t} ; \boldsymbol{e}^{j}(\boldsymbol{A}, i)$ denotes the unit eigenvector of a symmetric (and thus diagonalizable) matrix $\boldsymbol{A}$ (of size $[r \times r]$ ) corresponding to the eigenvalue $\lambda_{i}, i \in \mathcal{R} \triangleq\{1, \ldots r\}$, where $\lambda_{s}>\lambda_{s+1}$, $s \in \mathcal{R} \backslash\{r\}$, and $j \in\{-1,1\}$ denotes the sign ambiguity in the eigenvector formula; $\arctan _{2}(\boldsymbol{x})$,

\footnotetext{
${ }^{1}$ We will show however that for devising an efficient local-optimization algorithm a range of variability should be given; however the width of this range does not affect significantly the performance.
} 
$\boldsymbol{x} \in \mathbb{R}^{2}$, denotes the four-quadrant inverse tangent with argument $\frac{x_{1}}{x_{2}} ; P(\cdot)$ is used to denote probability mass functions (pmf) or probability density functions (pdf), while $P(\cdot \mid \cdot)$ is the corresponding conditional counterpart; $\mathcal{N}(\boldsymbol{\mu}, \boldsymbol{\Sigma})$ denotes a real normal distribution with mean vector $\boldsymbol{\mu}$ and covariance matrix $\boldsymbol{\Sigma}$; finally the symbols $\rightarrow, \ni, \sim$, and $\perp$ mean "maps to", "such that", "distributed as" and "orthogonal", respectively.

\section{System Model}

\section{A. Motion Models description}

The system model is described graphically in Fig. 3. We assume that the target is observed by the platform at $n$ time samples, i.e. $t \in \mathcal{T} \triangleq\left\{t_{1}, \ldots, t_{n}\right\}$; also we define the set of indices $\mathcal{I} \triangleq\{1, \ldots, n\}$. In the following we will explicitly list all the assumptions made, starting from the motion models of the platform and the target.

Assumption I: We assume that the target moves according to a constant velocity (CV) motion model [1]. For this reason we define $\boldsymbol{p}_{T}\left(t_{i}\right) \triangleq\left[\begin{array}{ll}\xi_{T}\left(t_{i}\right) & \eta_{T}\left(t_{i}\right)\end{array}\right]^{t}$ and $\boldsymbol{v}_{T} \triangleq\left[\begin{array}{ll}\dot{\xi}_{T} & \dot{\eta}_{T}\end{array}\right]^{t}$ as the position at $t_{i}$ and the (constant) velocity 2-D vector of the target; $\xi$ and $\eta$ are used to denote the east and north directions. Given the CV assumption, $\left\{\boldsymbol{p}_{T}\left(t_{1}\right), \boldsymbol{p}_{T}\left(t_{n}\right)\right\}$ uniquely define the state of the target at $t_{i} \in \mathcal{T}$. Therefore we stack them in $\boldsymbol{x}_{T} \triangleq\left[\begin{array}{ll}\boldsymbol{p}_{T}\left(t_{1}\right)^{t} & \boldsymbol{p}_{T}\left(t_{n}\right)^{t}\end{array}\right]^{t}$, which represents the true target state vector, unknown at the platform side. The target motion model has the explicit expression:

$$
\begin{aligned}
{\left[\begin{array}{c}
\xi_{T}\left(t_{i}\right) \\
\eta_{T}\left(t_{i}\right)
\end{array}\right] } & =\boldsymbol{p}_{T}\left(t_{1}\right)+\left(t_{i}-t_{1}\right) \cdot \boldsymbol{v}_{T} \\
& =\boldsymbol{p}_{T}\left(t_{1}\right)+\underbrace{\frac{\left(t_{i}-t_{1}\right)}{\left(t_{n}-t_{1}\right)}}_{\triangleq \alpha_{i}}\left[\boldsymbol{p}_{T}\left(t_{n}\right)-\boldsymbol{p}_{T}\left(t_{1}\right)\right], \quad t_{i} \in \mathcal{T}
\end{aligned}
$$

Assumption II: We assume a platform moving according to a "two-leg" motion model; this requirement not only ensures observability of the target from the platform point of view [23], [24], but also represents the easiest trajectory that can be followed by the platform. We denote $\boldsymbol{p}_{P}\left(t_{i}\right) \triangleq\left[\begin{array}{ll}\xi_{P}\left(t_{i}\right) & \eta_{P}\left(t_{i}\right)\end{array}\right]^{t}, \boldsymbol{v}_{P, 1} \triangleq\left[\begin{array}{ll}\dot{\xi}_{P, 1} & \dot{\eta}_{P, 1}\end{array}\right]^{t}$ and $\boldsymbol{v}_{P, 2} \triangleq\left[\begin{array}{ll}\dot{\xi}_{P, 2} & \dot{\eta}_{P, 2}\end{array}\right]^{t}$, as the position at $t_{i} \in \mathcal{T}$, first-leg and second-leg velocity vectors; also we group $\boldsymbol{x}_{P} \triangleq\left[\begin{array}{lll}\boldsymbol{p}_{P}\left(t_{1}\right)^{t} & \boldsymbol{v}_{P, 1}^{t} & \boldsymbol{v}_{P, 2}^{t}\end{array}\right]^{t}$ into the platform state vector, representing the unknowns at the target-friendly side. Note that $\boldsymbol{x}_{P}$ does not uniquely define the platform trajectory, since the turning time $t_{k}$ is also needed. 
Assumption III: Throughout this paper we will make the simplifying assumption that $t_{k}$ is known at the target-friendly side. In fact it is reasonable to assume that $t_{k}$ in practice will happen nearly the middle of the observation interval, i.e., $t_{k} \approx \frac{t_{n}-t_{1}}{2}$, in order to assure a good degree of observability ${ }^{2}$. This assumption will be relaxed in Sec. V, where a sensitivity analysis with respect to (w.r.t.) the timing-uncertainty on $t_{k}$ will be shown. Therefore, once $t_{k}$ is assumed to be known, the platform motion model is explicitly described as

$$
\left[\begin{array}{l}
\xi_{P}\left(t_{i}\right) \\
\eta_{P}\left(t_{i}\right)
\end{array}\right]= \begin{cases}\boldsymbol{p}_{P}\left(t_{1}\right)+\left(t_{i}-t_{1}\right) \boldsymbol{v}_{P, 1} & t_{i} \in \mathcal{T}, \ni t_{i}<t_{k} \\
\boldsymbol{p}_{P}\left(t_{1}\right)+\left(t_{k}-t_{1}\right) \boldsymbol{v}_{P, 1}+\left(t_{i}-t_{k}\right) \boldsymbol{v}_{P, 2} & t_{i} \in \mathcal{T}, \ni t_{i} \geq t_{k}\end{cases}
$$

Note that here the magnitudes of $\boldsymbol{v}_{P, 1}$ and $\boldsymbol{v}_{P, 2}$ are arbitrary. As shown later, unique identifiability of the platform trajectory (our goal) requires these magnitudes to be the same, i.e., the platform speed should be constant.

\section{B. ML-PDA statistical assumptions and formulation}

The statistical assumptions on the measurements are summarized as follows.

Assumption I: The bearing (true-originated from the target) measurement $\kappa_{i}$, collected by the platform at $t_{i}$, follows the model

$$
\kappa_{i}=\theta_{i}\left(\boldsymbol{x}_{T}, \boldsymbol{x}_{P}\right)+n_{i}=\arctan \left(\frac{\xi_{T}\left(t_{i}\right)-\xi_{P}\left(t_{i}\right)}{\eta_{T}\left(t_{i}\right)-\eta_{P}\left(t_{i}\right)}\right)+n_{i}
$$

where $\theta_{i}\left(\boldsymbol{x}_{T}, \boldsymbol{x}_{P}\right)$ denotes the noise-free bearing (we stress the dependence on both platform and target state vectors) and $n_{i} \sim \mathcal{N}\left(0, \sigma_{\theta}^{2}\right)$. For notational convenience we also define here the range $r_{i}\left(\boldsymbol{x}_{T}, \boldsymbol{x}_{P}\right)$ as

$$
r_{i}\left(\boldsymbol{x}_{T}, \boldsymbol{x}_{P}\right) \triangleq\left\|\boldsymbol{p}_{T}\left(t_{i}\right)-\boldsymbol{p}_{P}\left(t_{i}\right)\right\|_{2}
$$

Assumption II: We assume, as in realistic environments, that a passive sonar at $t_{i} \in \mathcal{T}$ collects a set of measurements $\boldsymbol{z}(i)$, due to clutter and non-perfect detection. More specifically, we have

$$
\boldsymbol{z}(i) \triangleq\left\{z_{j}(i)\right\}_{j=1}^{m_{i}}
$$

\footnotetext{
${ }^{2}$ In fact, a turn at the beginning or the end of the observation interval would result in a platform trajectory similar to a single leg (CV model), thus leading to a nearly singular FIM.
} 


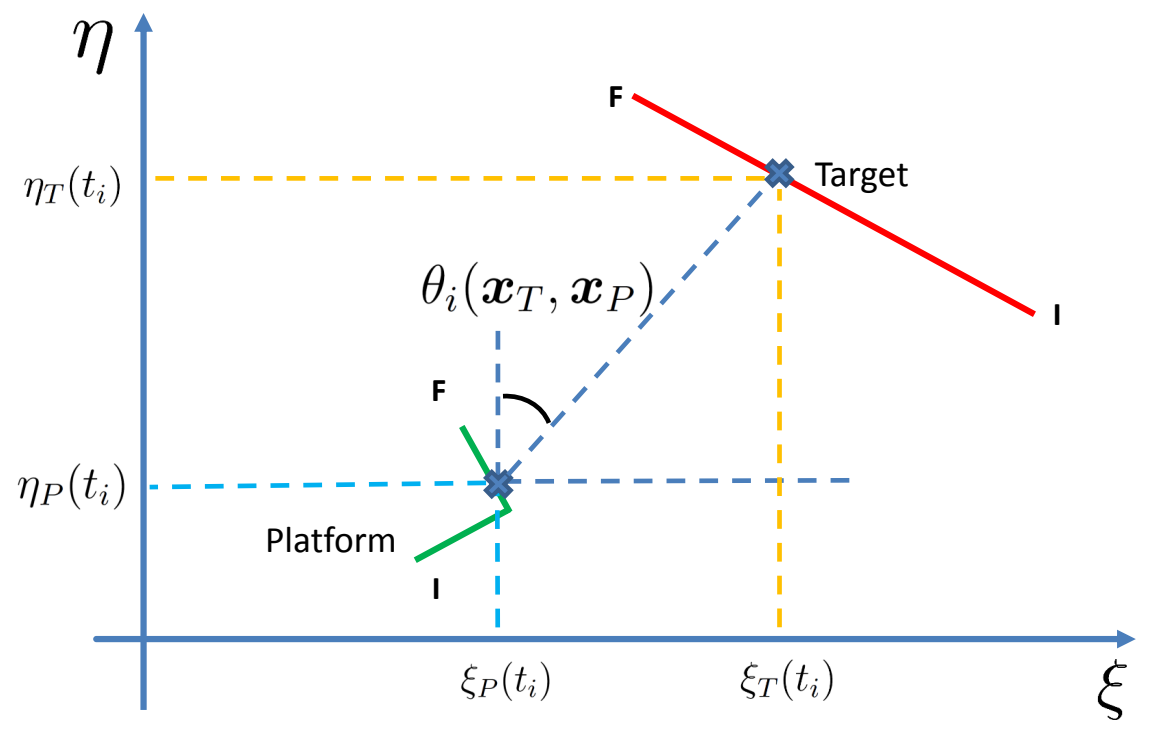

Figure 3: System model considered for our application. The crosses refer to the bearing measurement at snapshot $t_{i} \in \mathcal{T}$.

where $m_{i}$ denotes the number of collected measurements at $t_{i} \in \mathcal{T}$. The statistical assumptions over the set in Eq. (6) are: (i) the true measurement $\kappa_{i}$ can be detected at most only once, with probability $P_{D}$; (ii) the number of false measurements at $t_{i} \in \mathcal{T}$ follows a known probability mass function $\mu_{F}(\cdot)$, given by a Poisson law with known expected number of false alarms per unit of volume $\lambda$; therefore the false measurements are distributed uniformly and independently in the surveillance region (in the bearing space).

Assumption III: We assume conditional mutual independence among the sets of measurements, that is $P\left(\boldsymbol{z}\left(i_{1}\right), \boldsymbol{z}\left(i_{2}\right) \mid \boldsymbol{x}_{T}, \boldsymbol{x}_{P}\right)=P\left(\boldsymbol{z}\left(i_{1}\right) \mid \boldsymbol{x}_{T}, \boldsymbol{x}_{P}\right) P\left(\boldsymbol{z}\left(i_{2}\right) \mid \boldsymbol{x}_{T}, \boldsymbol{x}_{P}\right), \forall i_{1} \neq i_{2}$.

Under these assumptions and denoting $\breve{\boldsymbol{x}}_{T}$ (resp. $\breve{\boldsymbol{x}}_{P}$ ) as the true target (resp. platform) state 
vector, the ML-PDA estimate $\hat{\boldsymbol{x}}_{T}$ is obtained as

$$
\hat{\boldsymbol{x}}_{T} \triangleq \arg \max _{\boldsymbol{x}_{T}} \prod_{i=0}^{n} P\left(\boldsymbol{z}(i) \mid \boldsymbol{x}_{T}, \breve{\boldsymbol{x}}_{P}\right)
$$

where the likelihood of $\boldsymbol{x}_{T}$ is given by:

$$
\begin{aligned}
P\left(\boldsymbol{z}(i) \mid \boldsymbol{x}_{T}, \breve{\boldsymbol{x}}_{P}\right) & =u^{-m_{i}}\left(1-P_{D}\right) \mu_{F}\left(m_{i}\right) \\
& +\frac{u^{1-m_{i}} P_{D} \mu_{F}\left(m_{i}-1\right)}{m_{i}} \sum_{j=1}^{m_{i}} \frac{1}{\sqrt{2 \pi} \sigma_{\theta}} \times \exp \left(-\frac{1}{2}\left(\frac{z_{j}(i)-\theta_{i}\left(\boldsymbol{x}_{T}, \breve{\boldsymbol{x}}_{P}\right)}{\sigma_{\theta}}\right)^{2}\right)
\end{aligned}
$$

Note that $\breve{\boldsymbol{x}}_{P}$ is assumed known at the platform side. It was shown numerically in [17] that the covariance matrix of the ML-PDA estimator essentially attains the CRLB and therefore it can be regarded as an efficient estimator. For this reason, the covariance matrix is approximated by the inverse of the FIM given by

$$
\boldsymbol{J}\left(\boldsymbol{x}_{T}, \boldsymbol{x}_{P}, \alpha_{\theta}\right) \triangleq \alpha_{\theta} \underbrace{\sum_{i=0}^{n} \boldsymbol{\nabla}_{\boldsymbol{x}_{T}}\left(\theta_{i}\left(\boldsymbol{x}_{T}, \boldsymbol{x}_{P}\right)\right) \boldsymbol{\nabla}_{\boldsymbol{x}_{T}}^{t}\left(\theta_{i}\left(\boldsymbol{x}_{T}, \boldsymbol{x}_{P}\right)\right)}_{\triangleq \boldsymbol{J}_{u}\left(\boldsymbol{x}_{T}, \boldsymbol{x}_{P}\right)}
$$

where $\alpha_{\theta}$ is defined as ${ }^{3}$

$$
\alpha_{\theta} \triangleq \frac{q_{2}}{\sigma_{\theta}^{2}}
$$

with $q_{2}$ representing the IRF [17], [2]. Note that

$$
q_{2}=q_{2}\left(\lambda v_{g}, P_{D}, g\right)
$$

where $v_{g}$ and $g$ denote the volume of the validation region and the gating threshold, respectively. It can be shown, after some manipulations, that $\boldsymbol{\nabla}_{\boldsymbol{x}_{T}}\left(\theta_{i}\left(\boldsymbol{x}_{T}, \boldsymbol{x}_{P}\right)\right)$ has the explicit expression

$$
\boldsymbol{\nabla}_{\boldsymbol{x}_{T}}\left(\theta_{i}\left(\boldsymbol{x}_{T}, \boldsymbol{x}_{P}\right)\right)=\frac{1}{r_{i}\left(\boldsymbol{x}_{T}, \boldsymbol{x}_{P}\right)}\left[\begin{array}{c}
\left(1-\alpha_{i}\right) \cos \left(\theta_{i}\left(\boldsymbol{x}_{T}, \boldsymbol{x}_{P}\right)\right) \\
-\left(1-\alpha_{i}\right) \sin \left(\theta_{i}\left(\boldsymbol{x}_{T}, \boldsymbol{x}_{P}\right)\right) \\
\alpha_{i} \cos \left(\theta_{i}\left(\boldsymbol{x}_{T}, \boldsymbol{x}_{P}\right)\right) \\
-\alpha_{i} \sin \left(\theta_{i}\left(\boldsymbol{x}_{T}, \boldsymbol{x}_{P}\right)\right)
\end{array}\right]
$$

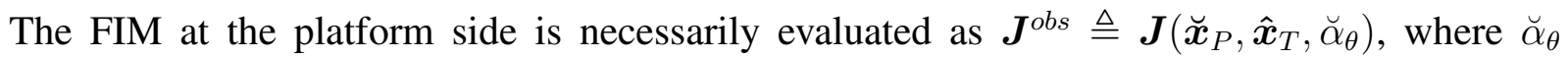
denotes the true $\alpha_{\theta}$, known at the platform side. Given the results of the estimation process at the platform side, that is $\left\{\hat{\boldsymbol{x}}_{T}, \boldsymbol{J}^{\text {obs }}\right\}$, our task can be summarized as follows.

\footnotetext{
${ }^{3}$ Note that $\alpha_{\theta}$ should not be confused with $\alpha_{i}=\frac{t_{i}-t_{1}}{t_{n}-t_{1}}$ defined in Eq. (2).
} 
We wish to identify the platform state, represented by $\boldsymbol{x}_{P}$, by observing only the estimation results of the ML-PDA, that is $\left\{\hat{\boldsymbol{x}}_{T}, \boldsymbol{J}^{\text {obs }}\right\}$. It is worth remarking that the unknowns of this deterministic problem are represented by $\left\{\breve{\boldsymbol{x}}_{P}, \breve{\alpha}_{\theta}\right\}$. In fact, even if $\breve{\alpha}_{\theta}$ does not contribute to specifying the platform trajectory, it has to be identified to solve this task.

The first important remark is that the identification problem is a function only of $\hat{\boldsymbol{x}}_{T}$ rather than the true trajectory $\breve{\boldsymbol{x}}_{T}$. This has an important consequence: the identification of the platform trajectory does not depend on the true target trajectory $\breve{\boldsymbol{x}}_{T}$; however we will show that due to the sensitivity w.r.t. the true platform parameters, a larger FIM matrix will lead to an easier identification in terms of local optimization routines.

\section{Objective Function Determination}

As a starting point of the identification problem, it would be natural to solve the non-linear equation

$$
\boldsymbol{J}\left(\hat{\boldsymbol{x}}_{T}, \boldsymbol{x}_{P}, \alpha_{\theta}\right)=\boldsymbol{J}^{\text {obs }}
$$

for the variables $\boldsymbol{x}_{P}$ and $\alpha_{\theta}$. However, as stated by the following proposition, we will show that this system is unobservable, since there exists an infinite number of solutions satisfying Eq. (13).

Proposition 1. If $\left\{\boldsymbol{x}_{P}^{*}, \alpha_{\theta}^{*}\right\}$ is a solution of (13), then each $\left\{\boldsymbol{x}_{P}^{\prime}, \alpha_{\theta}^{\prime}\right\}$ generated by the subspace

$$
\begin{aligned}
\left\{\boldsymbol{x}_{P}^{\prime}, \alpha_{\theta}^{\prime}\right\} & =\left\{\beta \boldsymbol{x}_{P}^{*}+(1-\beta) \overline{\boldsymbol{x}}_{E}, \beta^{2} \alpha_{\theta}^{*}\right\}, \quad \beta \in \mathbb{R} \\
\overline{\boldsymbol{x}}_{E} & \triangleq\left[\begin{array}{lll}
\hat{\boldsymbol{p}}_{T}\left(t_{1}\right)^{t} & \hat{\boldsymbol{v}}_{T}^{t} & \hat{\boldsymbol{v}}_{T}^{t}
\end{array}\right]^{t}
\end{aligned}
$$

is also a solution of (13).

Proof: The proof is given in Appendix A.

The above proposition states that no unambiguous identification of the platform trajectory $\breve{\boldsymbol{x}}_{P}$ is possible when the platform is following a trajectory according to Eq. (3) with different speed in each leg. The explanation of this is given by the fact that an affine combination (through $\beta$ ) of the platform and the (augmented) target state vector would produce the same FIM with an $\alpha_{\theta}$ scaled by $\beta^{2}$. At this point it is worth stressing the difference between this requirement and the target estimation, in which the platform maneuver is the only prerequisite to ensure observability of the target by the platform. 
However, when both legs of the platform trajectory are constrained to have the same speed $s$, that is

$$
s \triangleq\left\|\boldsymbol{v}_{P, 1}\right\|_{2}=\left\|\boldsymbol{v}_{P, 2}\right\|_{2}
$$

we can show that the subspace described by Eq. (14) violates constraint (16), if the specific condition

$$
\left(\boldsymbol{v}_{P, 1}-\boldsymbol{v}_{P, 2}\right)^{t} \hat{\boldsymbol{v}}_{T} \neq 0
$$

holds. The latter represents the condition under which the difference vector of the two legs velocities is orthogonal to the velocity vector of the target; such a trajectory would make the platform unobservable, even though the constraint in Eq. (16) is satisfied, and thus it should be intended as a stealthy trajectory achievable by the platform ${ }^{4}$. To demonstrate this, let us consider the squared speed of the two legs for a platform trajectory belonging to the subspace in Eq. (14):

$$
\begin{aligned}
& \left\|\boldsymbol{v}_{P, 1}^{\prime}\right\|_{2}^{2}=\beta^{2}\left\|\boldsymbol{v}_{P, 1}\right\|_{2}^{2}+(1-\beta)^{2}\left\|\hat{\boldsymbol{v}}_{T}\right\|_{2}^{2}+2 \beta(1-\beta) \hat{\boldsymbol{v}}_{T}^{t} \boldsymbol{v}_{P, 1} \\
& \left\|\boldsymbol{v}_{P, 2}^{\prime}\right\|_{2}^{2}=\beta^{2}\left\|\boldsymbol{v}_{P, 2}\right\|_{2}^{2}+(1-\beta)^{2}\left\|\hat{\boldsymbol{v}}_{T}\right\|_{2}^{2}+2 \beta(1-\beta) \hat{\boldsymbol{v}}_{T}^{t} \boldsymbol{v}_{P, 2}
\end{aligned}
$$

If we constrain the true platform trajectory to keep constant speed during the two legs, evaluating the difference $\left\|\boldsymbol{v}_{P, 1}^{\prime}\right\|_{2}^{2}-\left\|\boldsymbol{v}_{P, 2}^{\prime}\right\|_{2}^{2}$ leads to

$$
\left\|\boldsymbol{v}_{P, 1}^{\prime}\right\|_{2}^{2}-\left\|\boldsymbol{v}_{P, 2}^{\prime}\right\|_{2}^{2}=2 \beta(1-\beta) \hat{\boldsymbol{v}}_{T}^{t}\left(\boldsymbol{v}_{P, 1}-\boldsymbol{v}_{P, 2}\right)
$$

From inspection of Eq. (20), it is apparent that a platform described by Eq. (14) keeps a constant speed in the two legs only if: $(i) \beta=1$, i.e. the trajectory considered coincides with $\boldsymbol{x}_{P}^{*}$; $($ ii $)$ $\beta=0$, which represents a degenerate platform trajectory and thus it can be excluded; (iii) $\hat{\boldsymbol{v}}_{T} \perp\left(\boldsymbol{v}_{P, 1}-\boldsymbol{v}_{P, 2}\right)$. For this reason, under the assumptions in Eqs. (16) and (17), identification of the platform trajectory is possible. More specifically, the constraint in Eq. (16) represents a necessary condition for observability of the platform trajectory. On the basis of this constraint we define a new platform-state vector $\boldsymbol{x}_{P}^{s}$ (and we denote the true platform-state vector as $\breve{\boldsymbol{x}}_{P}^{s}$ )

\footnotetext{
${ }^{4}$ Obviously, design of such platform trajectory would require in advance the knowledge of the estimated target trajectory and thus it is not performable in practice.
} 
as follows

$$
\begin{aligned}
\boldsymbol{x}_{P}^{s} & \triangleq\left[\begin{array}{lllll}
\xi_{P}\left(t_{1}\right) & \eta_{P}\left(t_{1}\right) & s & \phi_{1} & \phi_{2}
\end{array}\right]^{t} \\
\phi_{i} & \triangleq \arctan _{2}\left(\boldsymbol{v}_{P, i}\right), \quad i \in\{1,2\}
\end{aligned}
$$

Thus Eq. (13) becomes

$$
\boldsymbol{J}\left(\boldsymbol{x}_{P}^{s}, \alpha_{\theta}\right)=\boldsymbol{J}^{o b s}
$$

with unknowns $\boldsymbol{x}_{P}^{s}$ and $\alpha_{\theta}$ (starting from here we drop the dependence on $\hat{\boldsymbol{x}}_{T}$ to keep the notation simple). In general, the non-linear system described by Eq. (23) can still admit multiple solutions, since there is no theoretical proof that the set of constraints in Eqs. (16) and (17) is also a sufficient condition for observability of $\left\{\boldsymbol{x}_{P}^{s}, \alpha_{\theta}\right\}$. This is because proving that $\left\{\boldsymbol{x}_{P}^{s}, \alpha_{\theta}\right\} \rightarrow$ $\boldsymbol{J}\left(\boldsymbol{x}_{P}^{s}, \alpha_{\theta}\right)$ is a one-to-one mapping is an extremely difficult task. Nonetheless, we will show, through simulations in Sec. $\mathrm{V}$, that this property seems to be satisfied and that $\boldsymbol{x}_{P}^{s}$ can be identified.

To solve Eq. (23) in an efficient way we consider the search for the minimum of the square of the Frobenius norm $\mathcal{F}\left(\boldsymbol{x}_{P}^{s}, \alpha_{\theta}\right)$, namely,

$$
\left\{\hat{\boldsymbol{x}}_{P}^{s}, \hat{\alpha}_{\theta}\right\}=\arg \min _{\left\{\boldsymbol{x}_{P}^{s}, \alpha_{\theta}\right\}} \underbrace{\left\|\boldsymbol{J}^{o b s}-\boldsymbol{J}\left(\boldsymbol{x}_{P}^{s}, \alpha_{\theta}\right)\right\|_{F}^{2}}_{\triangleq \mathcal{F}\left(\boldsymbol{x}_{P}^{s}, \alpha_{\theta}\right)}
$$

It is easy to see that the global minimum (corresponding to a zero value) of Eq. (24) corresponds to the solution of Eq. (23). Although the criterion of Eq. (24) appears as arbitrary (in fact other matrix distance norms can be considered), there is an important reason behind this choice: we will show in the following that $\mathcal{F}\left(\boldsymbol{x}_{P}^{s}, \alpha_{\theta}\right)$ (see (27)) can be expressed in terms of a weighted-square-distance, and an important property of weighted non-linear least squares problems can be exploited [20].

The first step to express Eq. (24) in terms of a convenient weighted-least squares problem is to search for independent entries of $\boldsymbol{J}\left(\boldsymbol{x}_{P}^{s}, \alpha_{\theta}\right)$. The following Lemma will be used.

Lemma 2. The FIM $\boldsymbol{J}\left(\boldsymbol{x}_{P}^{s}, \alpha_{\theta}\right)$ has only 9 independent entries.

Proof: The proof is given in Appendix B.

Exploiting Lemma 2, we can express Eq. (24) in terms of a weighted-square distance, as stated by the following proposition. 
Proposition 3. The square of the norm in Eq. (24) can be equivalently written in the form

$$
\mathcal{F}\left(\boldsymbol{x}_{P}^{s}, \alpha_{\theta}\right)=\left[\boldsymbol{j}^{\text {obs }}-\boldsymbol{j}\left(\boldsymbol{x}_{P}^{s}, \alpha_{\theta}\right)\right]^{t} \boldsymbol{W}\left[\boldsymbol{j}^{\text {obs }}-\boldsymbol{j}\left(\boldsymbol{x}_{P}^{s}, \alpha_{\theta}\right)\right]
$$

where $\boldsymbol{j}^{\text {obs }} \in \mathbb{R}^{9}$ and $\boldsymbol{j}\left(\boldsymbol{x}_{P}^{s}, \alpha_{\theta}\right) \in \mathbb{R}^{9}$ are obtained by stacking the independent components of $\boldsymbol{J}\left(\boldsymbol{x}_{P}^{s}, \alpha_{\theta}\right)$ and $\boldsymbol{J}^{\text {obs }}$, respectively and $\boldsymbol{W}$ is a diagonal weighting matrix, defined as follows:

$$
\boldsymbol{W} \triangleq \operatorname{diag}\left(\left[\begin{array}{lllllllll}
1 & 1 & 1 & 1 & 2 & 2 & 4 & 2 & 2
\end{array}\right]^{t}\right)
$$

Proof: The proof is given in Appendix C.

Note that $\boldsymbol{j}\left(\boldsymbol{x}_{P}^{s}, \alpha_{\theta}\right)$ retains the same factorization as $\boldsymbol{J}\left(\boldsymbol{x}_{P}^{s}, \alpha_{\theta}\right)$, that is, $\boldsymbol{j}\left(\boldsymbol{x}_{P}^{s}, \alpha_{\theta}\right)=\alpha_{\theta} \boldsymbol{j}_{u}\left(\boldsymbol{x}_{P}^{s}\right)$, where $\boldsymbol{j}_{u}\left(\boldsymbol{x}_{P}^{s}\right)$ is defined accordingly to Eq. (9). Hence Eq. (25) can be rewritten as

$$
\mathcal{F}\left(\boldsymbol{x}_{P}^{s}, \alpha_{\theta}\right)=\left[\boldsymbol{j}^{o b s}-\alpha_{\theta} \boldsymbol{j}_{u}\left(\boldsymbol{x}_{P}^{s}\right)\right]^{t} \boldsymbol{W}\left[\boldsymbol{j}^{o b s}-\alpha_{\theta} \boldsymbol{j}_{u}\left(\boldsymbol{x}_{P}^{s}\right)\right]
$$

Note that the minimization of the objective function in Eq. (27) is in the standard form of non-linear weighted least squares [20]. Additionally in this case the non-linear weighted square distance is linear in some of the parameters to be estimated, in this case $\alpha_{\theta}$, and thus this non-linear problem can be solved in a reduced dimension space; the details are given by the following proposition.

Proposition 4. The minimization in the 6-dimensional space of the objective $\mathcal{F}\left(\boldsymbol{x}_{P}^{s}, \alpha_{\theta}\right)$ is equivalent to maximization of the objective $\mathcal{G}\left(\boldsymbol{x}_{P}^{s}\right)$, defined as

$$
\begin{aligned}
\mathcal{G}\left(\boldsymbol{x}_{P}^{s}\right) & \triangleq\left\langle\boldsymbol{j}^{o b s}, \boldsymbol{c}\left(\boldsymbol{x}_{P}^{s}\right)\right\rangle^{2} \\
\boldsymbol{c}\left(\boldsymbol{x}_{P}^{s}\right) & \triangleq \frac{\boldsymbol{W} \boldsymbol{j}_{u}\left(\boldsymbol{x}_{P}^{s}\right)}{\left[\boldsymbol{j}_{u}\left(\boldsymbol{x}_{P}^{s}\right)^{t} \boldsymbol{W} \boldsymbol{j}_{u}\left(\boldsymbol{x}_{P}^{s}\right)\right]^{1 / 2}}
\end{aligned}
$$

Proof: The proof is given in Appendix D.

The maximization of $\mathcal{G}\left(\boldsymbol{x}_{P}^{s}\right)$ has the advantage of reducing the search space from $\mathbb{R}^{6}$ to $\mathbb{R}^{5}$. The exclusion of $\alpha_{\theta}$ also allows a search only in the subspace of variables determining the platform trajectory. At this point some observations on the objective function in Eq. (73) are in order:

- The function $\mathcal{G}\left(\boldsymbol{x}_{P}^{s}\right)$ is highly non-linear in $\boldsymbol{x}_{P}^{s}$, therefore no closed form solution to optimization of Eq. (28) exists; thus numerical optimization procedures need to be used; 
- Local optimization routines can get stuck in local maxima or other (non-equilibrium) points ${ }^{5}$. An important issue in local optimization routines that determines their success, is the choice of a good initial guess. In the following we will suggest a good start, based on the FIM and platform-target geometry.

\section{IV. “GEOMETRY-DRIVEN” FIM-AIDED INITIAL ESTIMATE CHOICE}

In this section we show how a good initial estimate, denoted as $\hat{\boldsymbol{x}}_{P}^{s, 0}$, can be chosen to help the convergence of the local optimization routines. To accomplish this task define

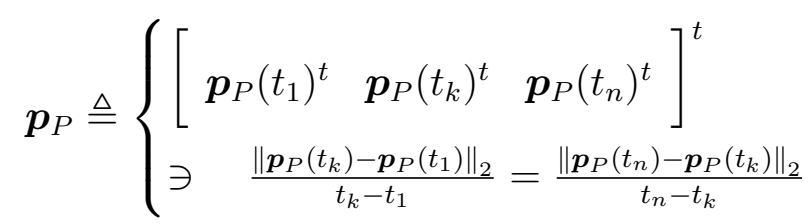

Since it is easy to show that there is a one-to-one mapping between $\boldsymbol{p}_{P}$ and $\boldsymbol{x}_{P}^{s}$, we will search for a $\hat{\boldsymbol{p}}_{P}^{0}$ close to $\breve{\boldsymbol{p}}_{P}$ (even though the latter is not known), as the equivalent input to the local optimization routine. For this reason, we first will find good approximations $\hat{\boldsymbol{p}}_{P}^{0}\left(t_{1}\right)$ and $\hat{\boldsymbol{p}}_{P}^{0}\left(t_{n}\right)$. After this, we will give the details on how to find $\hat{\boldsymbol{p}}_{P}^{0}\left(t_{k}\right)$, under the constraint of Eq. (30). The following considerations are based on the assumption that $\breve{\alpha}_{\theta}$ is known; at the end of the section we will remove this restriction. In the following, for the sake of simplicity, we will use the short-hand notations $r_{i} \triangleq r_{i}\left(\hat{\boldsymbol{x}}_{T}, \breve{\boldsymbol{x}}_{P}^{s}\right)$ for the range, $\theta_{i} \triangleq \theta_{i}\left(\hat{\boldsymbol{x}}_{T}, \breve{\boldsymbol{x}}_{P}^{s}\right)$ for the bearing, $\boldsymbol{i}_{i} \triangleq\left[\sin \left(\theta_{i}\right) \cos \left(\theta_{i}\right)\right]^{t}$ for the bearing unit vector, and we will drop the zero superscript in $\hat{\boldsymbol{p}}_{P}^{0}$ and $\hat{\boldsymbol{p}}_{P}^{0}\left(t_{i}\right)$.

\section{A. Choice of $\hat{\boldsymbol{p}}_{P}\left(t_{1}\right)$ and $\hat{\boldsymbol{p}}_{P}\left(t_{n}\right)$}

It can be easily shown that $\left\{\breve{\boldsymbol{p}}_{P}\left(t_{1}\right), \breve{\boldsymbol{p}}_{P}\left(t_{n}\right)\right\}$ can be expressed in the form:

$$
\begin{aligned}
& \breve{\boldsymbol{p}}_{P}\left(t_{1}\right)=\hat{\boldsymbol{p}}_{T}\left(t_{1}\right)+r_{1} \boldsymbol{i}_{1} \\
& \breve{\boldsymbol{p}}_{P}\left(t_{n}\right)=\hat{\boldsymbol{p}}_{T}\left(t_{n}\right)+r_{n} \boldsymbol{i}_{n}
\end{aligned}
$$

In order to obtain good estimates $\left\{\hat{\boldsymbol{p}}_{P}\left(t_{1}\right), \hat{\boldsymbol{p}}_{P}\left(t_{n}\right)\right\}$ we need to find good approximations $\left\{\hat{r}_{1}, \hat{r}_{n}\right\}$ and $\left\{\hat{\boldsymbol{i}}_{1}, \hat{\boldsymbol{i}}_{n}\right\}$. These issues can be tackled separately.

\footnotetext{
${ }^{5}$ In fact, several local optimization routines are not based on the evaluation of the gradient vector of the objective function at each iteration, thus a "null gradient" condition is not ensured when a stopping condition is met.
} 
Choice of $\left\{\hat{r}_{1}, \hat{r}_{n}\right\}$ : A coarse approximation of the ranges $r_{1}$ and $r_{n}$ is given by:

$$
\hat{r}_{1} \triangleq \sqrt{\frac{\breve{\alpha}_{\theta} \sum_{i=1}^{n}\left(1-\alpha_{i}\right)^{2}}{\operatorname{tr}\left(\boldsymbol{J}^{o b s}[1,1]\right)} ;} \quad \hat{r}_{n} \triangleq \sqrt{\frac{\breve{\alpha}_{\theta} \sum_{i=1}^{n} \alpha_{i}{ }^{2}}{\operatorname{tr}\left(\boldsymbol{J}^{o b s}[2,2]\right)}}
$$

where $\boldsymbol{J}^{o b s}[m, n]$ represents the $(m, n)$ th $[2 \times 2]$ block matrix of $\boldsymbol{J}^{\text {obs }}$, whose explicit expression in block form (exploiting the derivation in Appendix B) is given as follows:

$$
\begin{aligned}
\boldsymbol{J}^{o b s} & =\left[\begin{array}{cc}
\boldsymbol{J}^{o b s}[1,1] & \boldsymbol{J}^{o b s}[1,2] \\
\boldsymbol{J}^{o b s}[2,1] & \boldsymbol{J}^{o b s}[2,2]
\end{array}\right] \\
& =\alpha_{\theta}\left[\begin{array}{cc}
\sum_{i=1}^{n}\left(1-\alpha_{i}\right)^{2} \breve{\boldsymbol{y}}_{i} \breve{\boldsymbol{y}}_{i}^{t} & \sum_{i=1}^{n} \alpha_{i}\left(1-\alpha_{i}\right) \breve{\boldsymbol{y}}_{i} \breve{\boldsymbol{y}}_{i}^{t} \\
\sum_{i=1}^{n} \alpha_{i}\left(1-\alpha_{i}\right) \breve{\boldsymbol{y}}_{i} \breve{\boldsymbol{y}}_{i}^{t} & \sum_{i=1}^{n} \alpha_{i} \breve{\boldsymbol{y}}_{i} \breve{\boldsymbol{y}}_{i}^{t}
\end{array}\right]
\end{aligned}
$$

where

$$
\breve{\boldsymbol{y}}_{i} \triangleq \frac{1}{r_{i}\left(\hat{\boldsymbol{x}}_{T}, \breve{\boldsymbol{x}}_{P}^{s}\right)}\left[\cos \left(\theta_{i}\left(\hat{\boldsymbol{x}}_{T}, \breve{\boldsymbol{x}}_{P}^{s}\right)\right)-\sin \left(\theta_{i}\left(\hat{\boldsymbol{x}}_{T}, \breve{\boldsymbol{x}}_{P}^{s}\right)\right)\right]^{t}
$$

The term $\operatorname{tr}\left(\boldsymbol{J}^{o b s}[1,1]\right)$ (resp. $\left.\operatorname{tr}\left(\boldsymbol{J}^{o b s}[2,2]\right)\right)$ represents the sum of the eigenvalues of the FIM of $\boldsymbol{p}_{T}\left(t_{1}\right)$ (resp. $\boldsymbol{p}_{T}\left(t_{n}\right)$ ), that is, the sum of the square of the semiaxes of the corresponding ellipse. The terms in the numerators of Eq. (33) are correction factors which avoid biased estimates $\hat{r}_{1}$ and $\hat{r}_{n}$. The derivation of Eq. (33) is given in Appendix E.

It is worth remarking that Eq. (33) represents a rough approximation of $\left\{r_{1}, r_{n}\right\}$; however, as we will show in Sec. V, the accuracy obtained is sufficient to determine values close to $\breve{\boldsymbol{p}}_{P}\left(t_{1}\right)$ and $\breve{\boldsymbol{p}}_{P}\left(t_{n}\right)$ in all the practical scenarios considered.

Choice of $\left\{\hat{\boldsymbol{i}}_{1}, \hat{\boldsymbol{i}}_{n}\right\}$ : By defining $\boldsymbol{C}^{o b s} \triangleq\left(\boldsymbol{J}^{\text {obs }}\right)^{-1}$ and denoting $\boldsymbol{C}^{\text {obs }}[\ell, m]$ as the $(\ell, m)$ th $[2 \times 2]$ block matrix of $\boldsymbol{C}^{\text {obs }}$ we have that:

$$
\hat{\boldsymbol{i}}_{1}^{p} \triangleq \boldsymbol{e}^{p}\left(\boldsymbol{C}^{o b s}[1,1] ; 1\right), \quad \hat{\boldsymbol{i}}_{n}^{q} \triangleq \boldsymbol{e}^{q}\left(\boldsymbol{C}^{o b s}[2,2] ; 1\right) ; \quad p, q \in\{-1,1\}
$$

Note that $\boldsymbol{C}^{o b s}[1,1]$ (resp. $\boldsymbol{C}^{o b s}[2,2]$ ) is a lower bound of the covariance matrix of any unbiased estimator of $\boldsymbol{p}_{T}\left(t_{1}\right)$ (resp. $\boldsymbol{p}_{T}\left(t_{n}\right)$ ) and thus the eigenvector corresponding to the largest eigenvalue represents the axis of maximum uncertainty, i.e., the one along the range between the platform and the target. The indices $\{p, q\}$ underline the incomplete information about $\left\{\boldsymbol{i}_{1}, \boldsymbol{i}_{n}\right\}$ contained in the FIM, which intrinsically leads to a sign ambiguity (because if $e$ is an eigenvector, so is $-e)$. The derivation of Eq. (37) is given in Appendix F. 

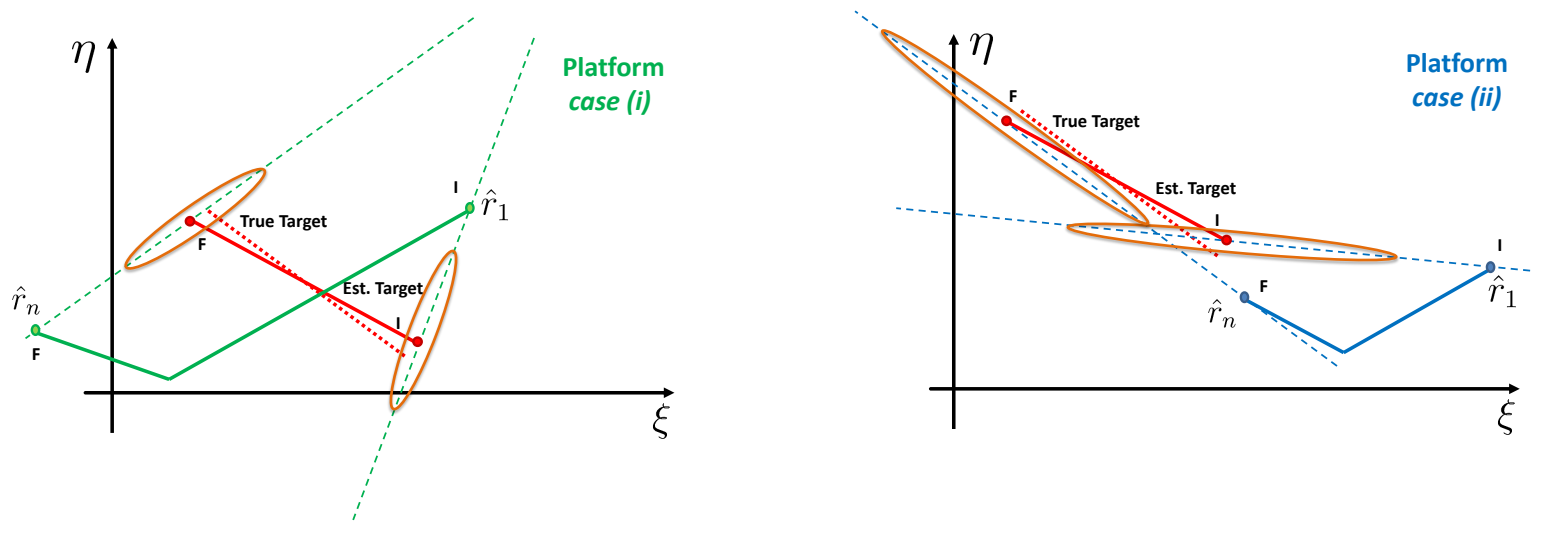

Figure 4: Graphical description of Eq. (38): cases (i) and (ii) satisfying it.

It is apparent that the sign ambiguity in Eq. (37) would lead to four possible pairs $\left\{\hat{\boldsymbol{i}}_{1}^{p}, \hat{\boldsymbol{i}}_{n}^{q}\right\}$. However, in practical scenarios the sign-ambiguity leads to only two pairs if we assume that the following vector equation has no solution:

$$
\zeta \cdot \hat{\boldsymbol{p}}_{T}\left(t_{1}\right)+(1-\zeta) \cdot \hat{\boldsymbol{p}}_{T}\left(t_{n}\right)=\boldsymbol{p}_{P}\left(t_{i}\right) ; \quad \forall t_{i} \in \mathcal{T}, \zeta \in \mathbb{R}
$$

Eq. (38) admits solutions only in the following $\operatorname{cases}^{6}$ : $(i)$ the platform trajectory and the target trajectory have at least one crossing point; (ii) the platform is crossing the line representing the direction of the CV target trajectory, determined by its velocity vector $\hat{\boldsymbol{v}}_{T}$. These scenarios are graphically depicted in Fig. 4. While the former case represents a totally unrealistic scenario (the platform and the target would be too near), it can be shown that the latter is of little interest, since such a platform trajectory would lead to poor observability of the target [24].

Therefore the only two admissible pairs are obtained as follows: let us define a vector $\{\boldsymbol{u} \in$ $\left.\mathbb{R}^{2}: \boldsymbol{u} \perp \hat{\boldsymbol{v}}_{T},\|\boldsymbol{u}\|_{2}=1\right\}$ and take the pairs $\left\{\hat{\boldsymbol{i}}_{1}^{p}, \hat{\boldsymbol{i}}_{n}^{q}\right\}$ satisfying

$$
\operatorname{sign}\left\langle\hat{\boldsymbol{i}}_{1}^{p}, \boldsymbol{u}\right\rangle=\operatorname{sign}\left\langle\hat{\boldsymbol{i}}_{n}^{q}, \boldsymbol{u}\right\rangle, \quad p, q \in\{-1,1\}
$$

In the following we denote as $\left\{\hat{\boldsymbol{i}}_{1}^{g}, \hat{\boldsymbol{i}}_{n}^{g}\right\}, g \in\{1,2\}$, the resulting two pairs. Thus, exploiting Eqs.

\footnotetext{
${ }^{6}$ Although Eq. (38) refers to $\hat{\boldsymbol{p}}_{T}\left(t_{i}\right)$, the same applies to $\boldsymbol{p}_{T}\left(t_{i}\right)$ under the assumption that the platform obtains a reasonably "confident" estimate of the target.
} 
(33), (37) and (39) we obtain:

$$
\begin{array}{ll}
\hat{\boldsymbol{p}}_{P}^{g}\left(t_{1}\right)=\hat{\boldsymbol{p}}_{T}\left(t_{1}\right)+\hat{r}_{1} \cdot \hat{\boldsymbol{i}}_{1}^{g}, & g \in\{1,2\} \\
\hat{\boldsymbol{p}}_{P}^{g}\left(t_{n}\right)=\hat{\boldsymbol{p}}_{T}\left(t_{n}\right)+\hat{r}_{n} \cdot \hat{\boldsymbol{i}}_{n}^{g}, & g \in\{1,2\}
\end{array}
$$

B. Choice of turning position vector $\hat{\boldsymbol{p}}_{P}\left(t_{k}\right)$

As pointed out previously, Eqs. (40) and (41) define two pairs $\left\{\hat{\boldsymbol{p}}_{P}^{g}\left(t_{1}\right), \hat{\boldsymbol{p}}_{P}^{g}\left(t_{n}\right)\right\}, g \in\{1,2\}$. Hence for each $\left\{\hat{\boldsymbol{p}}_{P}^{g}\left(t_{1}\right), \hat{\boldsymbol{p}}_{P}^{g}\left(t_{n}\right)\right\}$ a corresponding $\hat{\boldsymbol{p}}_{P}^{g}\left(t_{k}\right)$ needs to be computed. In this case it can be shown that $\hat{\boldsymbol{p}}_{P}^{g}\left(t_{k}\right)$ cannot be chosen only relying on the geometric properties of the FIM, as opposed to $\hat{\boldsymbol{p}}_{P}\left(t_{1}\right)$ and $\hat{\boldsymbol{p}}_{P}\left(t_{n}\right)$. Rather, a $\pm \frac{\pi}{2}$ platform turn initial assumption is made and the turn-sign ambiguity is solved exploiting the coarse information in the FIM. By defining $t_{m} \triangleq \arg \min _{t_{i} \in \mathcal{T}}\left\|t_{i}-\frac{t_{n}-t_{1}}{2}\right\|_{2}$, such a vector is obtained as (we drop the superscript $g$ )

$$
\begin{aligned}
\hat{\boldsymbol{p}}_{P}\left(t_{k}\right) & =\boldsymbol{\rho}_{q}\left(t_{k}\right) \\
\boldsymbol{\rho}_{\ell}\left(t_{k}\right) & \triangleq \hat{\boldsymbol{p}}_{P}\left(t_{1}\right)+\frac{\left(t_{k}-t_{1}\right)}{\left(t_{n}-t_{k}\right)}\left\|\hat{\boldsymbol{p}}_{P}\left(t_{n}\right)-\hat{\boldsymbol{p}}_{P}\left(t_{1}\right)\right\|_{2} \cos (\nu)\left[\begin{array}{c}
\sin \left(\psi_{\ell}\right) \\
\cos \left(\psi_{\ell}\right)
\end{array}\right] \\
\psi_{\ell} & \triangleq \arctan _{2}\left(\hat{\boldsymbol{p}}_{P}\left(t_{n}\right)-\hat{\boldsymbol{p}}_{P}\left(t_{1}\right)\right)+\ell \cdot\left(\frac{\pi}{2}-\nu\right) \\
\nu & \triangleq \arctan \left(\frac{t_{k}-t_{1}}{t_{n}-t_{k}}\right) \\
q & \triangleq \arg \min _{\ell \in\{-1,1\}}\left\|\tilde{\boldsymbol{p}}_{P}\left(t_{m}\right)-\boldsymbol{\rho}_{\ell}\left(t_{m}\right)\right\|_{2}
\end{aligned}
$$

where $\boldsymbol{\rho}_{\ell}\left(t_{m}\right)$ is the position vector at $t_{m}$ of the two-leg trajectory described by $\left\{\hat{\boldsymbol{p}}_{P}\left(t_{1}\right), \boldsymbol{\rho}_{\ell}\left(t_{k}\right), \hat{\boldsymbol{p}}_{P}\left(t_{n}\right)\right\}$, and $\tilde{\boldsymbol{p}}_{P}\left(t_{m}\right)$ is given by

$$
\begin{aligned}
\tilde{\boldsymbol{p}}_{P}\left(t_{m}\right) & \triangleq \hat{\boldsymbol{p}}_{T}\left(t_{m}\right)+\tilde{r}_{m} \cdot \tilde{\boldsymbol{i}}_{m} \\
\tilde{r}_{m} & \triangleq \sqrt{\frac{\breve{\alpha}_{\theta} \sum_{i=1}^{n} \alpha_{i}\left(1-\alpha_{i}\right)}{\operatorname{tr}\left(\boldsymbol{J}^{o b s}[1,2]\right)}} \\
\tilde{\boldsymbol{i}}_{m} & \triangleq \boldsymbol{e}^{q}\left(\boldsymbol{J}^{o b s}[1,2] ; 1\right) \\
q & \ni \operatorname{sign}\left\langle\boldsymbol{e}^{j}\left(\boldsymbol{J}^{o b s}[1,2] ; 1\right), \boldsymbol{u}\right\rangle=\operatorname{sign}\left\langle\hat{\boldsymbol{i}}_{1}, \boldsymbol{u}\right\rangle, \quad j \in\{-1,1\} .
\end{aligned}
$$

The complete derivation of the selection of $\hat{\boldsymbol{p}}_{p}^{g}\left(t_{k}\right)$ is given in Appendix G. 


\section{Remarks on $\alpha_{\theta}$}

The presented method computes $\hat{\boldsymbol{p}}_{P}^{g}=\left[\begin{array}{lll}\hat{\boldsymbol{p}}_{P}^{g}\left(t_{1}\right)^{t} & \hat{\boldsymbol{p}}_{P}^{g}\left(t_{k}\right)^{t} \quad \hat{\boldsymbol{p}}_{P}^{g}\left(t_{n}\right)^{t}\end{array}\right]^{t}, g \in\{1,2\}$, under the assumption that $\breve{\alpha}_{\theta}$ is known at the target-friendly side. However, since $\breve{\alpha}_{\theta}$ is known exactly only at the platform side, we will replace $\breve{\alpha}_{\theta}$ with the variable $\alpha_{\theta}$ in Eq. (33), thus leading to $\hat{\boldsymbol{p}}_{P}^{g}=\hat{\boldsymbol{p}}_{P}^{g}\left(\alpha_{\theta}\right)$, i.e., a continuum of initial guesses. Therefore, in order to obtain a (small) finite set of initial guesses we apply the following steps:

1) define a uniform grid of $N_{\theta}$ values on $\alpha_{\theta}$, constrained in the interval $\left[\alpha_{\theta, \min }, \alpha_{\theta, \max }\right]^{7}$ and, after denoting the $m$-th value as $\alpha_{\theta}[m]=\alpha_{\theta, \min }+\frac{m-1}{N_{\theta}-1}\left(\alpha_{\theta, \max }-\alpha_{\theta, \min }\right), m \in \mathcal{S}_{\theta} \triangleq$ $\left\{1, \ldots, N_{\theta}\right\}$, we compute $\hat{\boldsymbol{p}}_{P}^{g}\left(\alpha_{\theta}[m]\right), g \in\{1,2\}, m \in \mathcal{S}_{\theta}$;

2) split up the set $\hat{\boldsymbol{p}}_{P}^{g}\left(\alpha_{\theta}[m]\right), m \in \mathcal{S}_{\theta}, g \in\{-1,1\}$ into three subsets, corresponding to zones $(a),(b)$ and $(c)$, defined as follows ${ }^{8}:(i)$ we split $\hat{\boldsymbol{p}}_{P}^{g}\left(\alpha_{\theta}[m]\right)$ into two sets $\hat{\boldsymbol{p}}_{P}^{g=1}\left(\alpha_{\theta}[m]\right)$ and $\hat{\boldsymbol{p}}_{P}^{g=-1}\left(\alpha_{\theta}[m]\right) ;(i i)$ by defining $\Gamma_{m, g} \triangleq \arctan _{2}\left(\hat{\boldsymbol{p}}_{P}^{g}\left(t_{n}, \alpha_{\theta}[m]\right)-\hat{\boldsymbol{p}}_{P}^{g}\left(t_{1}, \alpha_{\theta}[m]\right)\right)$ we seek for $\left\{m^{*}, g^{*}\right\} \ni \operatorname{sign}\left(\Gamma_{m^{*}, g^{*}}\right) \neq \operatorname{sign}\left(\Gamma_{\left(m^{*}+1\right), g^{*}}\right)$; (iii) we split the set corresponding to $g^{*}$ as $\hat{\boldsymbol{p}}_{P}^{g^{*}}\left(\alpha_{\theta}\left[1: m^{*}\right]\right)$ and $\hat{\boldsymbol{p}}_{P}^{g^{*}}\left(\alpha_{\theta}\left[\left(m^{*}+1\right): N_{\theta}\right]\right)$;

3) choose, from each defined subset, the guess corresponding to the maximum of Eq. (28), and denote the obtained triple as $\left\{\hat{\boldsymbol{p}}_{P}^{A}, \hat{\boldsymbol{p}}_{P}^{B}, \hat{\boldsymbol{p}}_{P}^{C}\right\}$, which is fed (possibly in parallel) to a local optimization routine.

A good choice of $N_{\theta}$ can be obtained as follows. We first observe that the worst-case error in grid sampling of $\alpha_{\theta}$ is given by $\Delta \varepsilon_{\theta}=\frac{\left(\alpha_{\theta, \max }-\alpha_{\theta, \min }\right)}{2 \cdot\left(N_{\theta}-1\right)}$; such error is negligible in Eq. (33) when $\breve{\alpha}_{\theta} \gg \Delta \varepsilon_{\theta}$, thus leading to $N_{\theta} \gg \frac{\alpha_{\theta, \max }-\alpha_{\theta, \min }}{2 \breve{\alpha}_{\theta}}+1$. However, since $\breve{\alpha}_{\theta}$ is not known, we can consider the conservative inequality $\alpha_{\theta, \min } \gg \Delta \varepsilon_{\theta}$, which leads to $N_{\theta} \gg \frac{1}{2} \frac{\alpha_{\theta, \min }+\alpha_{\theta, \max }}{\alpha_{\theta, \min }}$.

\section{Simulation Results}

In this section we consider two scenarios, taken from [1], to corroborate the theoretical results presented and show the performance of the geometry-driven initial guess procedure. In both scenarios $(i)$ and $(i i)$ we assume the same $\hat{\boldsymbol{x}}_{T}$ (since the absolute position is irrelevant, only the relative geometry), while we consider two different $\breve{\boldsymbol{x}}_{P}^{s}$ (see Table I). In Table I we report the

\footnotetext{
${ }^{7}$ The values $\left\{\alpha_{\theta, \min }, \alpha_{\theta, \max }\right\}$ are obtained automatically, once a reasonable range is given for parameters concurring with the definition of $\alpha_{\theta}$ (e.g. $\sigma_{\theta}$ ); an example will be given in Section V.
}

${ }^{8}$ Note that the definition of the zones is arbitrary. 
Table I: Parameters known at the platform side.

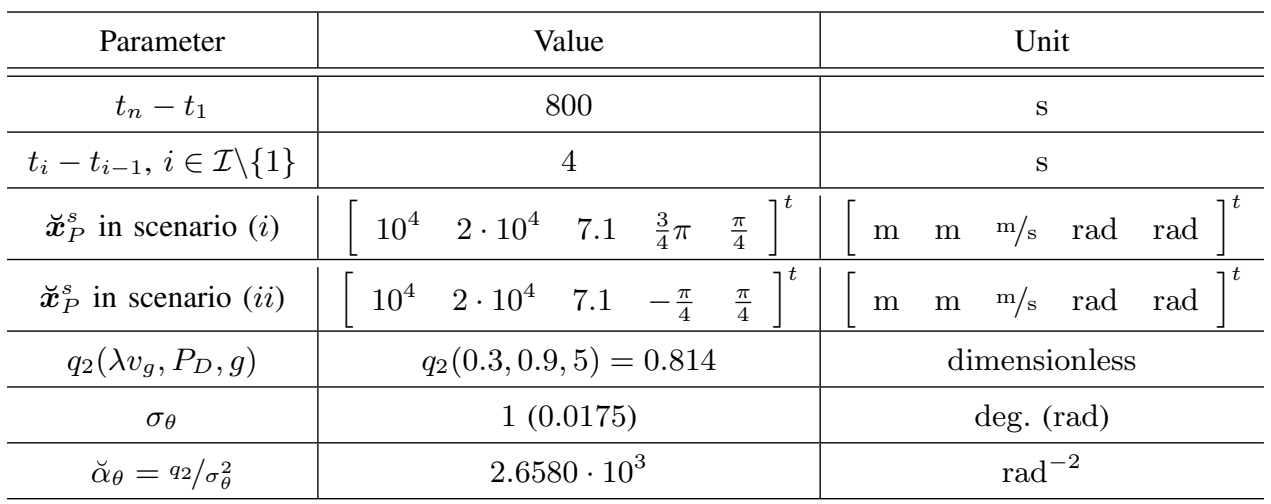

Table II: Parameters known at the target-friendly side.

\begin{tabular}{|c|c|c|}
\hline Parameter & Value & Unit \\
\hline$t_{n}-t_{1}$ & 800 & s \\
\hline$t_{i}-t_{i-1}, i \in \mathcal{I} \backslash\{1\}$ & 4 & $\mathrm{~s}$ \\
\hline$\hat{\boldsymbol{x}}_{T}$ & {$\left[\begin{array}{llll}15 \times 10^{3} & 35 \times 10^{3} & -10 & 5\end{array}\right]^{t}$} & {$\left[\begin{array}{llll}\mathrm{m} & \mathrm{m} & \mathrm{m} / \mathrm{s} & \mathrm{m} / \mathrm{s}\end{array}\right]^{t}$} \\
\hline$k$ & 101 & dimensionless \\
\hline$q_{2} *$ & $\in[0.652,0.982]$ & dimensionless \\
\hline$\sigma_{\theta} *$ & $\in[1,2](\in[0.0175,0.0350])$ & deg. (rad) \\
\hline$\alpha_{\theta} *$ & $\in[532.2449,3206.5]$ & $\mathrm{rad}^{-2}$ \\
\hline
\end{tabular}

list of parameters known at the platform side; it is worth noting that $\breve{\alpha}_{\theta}$ is completely specified by $q_{2}$ (obtained as in [17, Table II]) and $\sigma_{\theta}$.

In Table II we report the list of parameters known at the target-friendly side, after intercepting the ML-PDA estimates. The interval $\left[\alpha_{\theta, \min }, \alpha_{\theta, \max }\right]$ (needed to obtain the initial guesses in the three zones) is obtained as follows. We assume that the target-friendly entity possesses the coarse information $\sigma_{\theta} \in[1,2]^{\circ}$ and $q_{2} \in[0.652,0.982]$ (under the assumptions ${ }^{9}$ that $\lambda v_{g} \in[0.1,0.5]$, $P_{D} \in[0.8,1], g=5$, cf. [17, Table II]), thus leading to $\alpha_{\theta} \in[532.2449,3206.5] \mathrm{rad}^{-2}$.

The guesses $\left\{\hat{\boldsymbol{p}}_{P}^{A}, \hat{\boldsymbol{p}}_{P}^{B}, \hat{\boldsymbol{p}}_{P}^{C}\right\}$, obtained with the approach described in Sec. IV and setting $N_{\theta}=$

\footnotetext{
${ }^{9}$ Note that in Table II the asterisk indicates that those parameters are only needed to compute wise initial guesses for the local optimization procedure, but not for the maximization of Eq. (28).
} 
$5^{10}$, are given as input to the Nelder-Mead simplex method [22], which seeks for a local maximum of the objective function in Eq. (28). This local optimization routine has been chosen because it is a derivative-free method, as opposed to Newtonian and quasi-Newtonian local optimization routines. Such a choice avoids the evaluation of the Jacobian matrix (and also of the Hessian matrix, in the Newtonian approaches), which is composed of 45 entries, thus requiring extensive computations.

Fig. 5 presents a plot of the platform and target trajectories for scenario (i), while Fig. 9 shows them for scenario (ii). It is worth noting that scenario $(i)$ represents a low-observability case, while scenario (ii) has good observability, as shown through the $95 \%$ confidence ellipses of $\hat{\boldsymbol{p}}_{T}\left(t_{1}\right)$ and $\hat{\boldsymbol{p}}_{T}\left(t_{n}\right)$ in Figs. 5 and 9.

In Fig. 6 (resp. Fig. 10) we show the initial estimates corresponding to zones $(a),(b)$ and (c) (defined in step 2) of Subsec. IV-C) of the platform trajectory (28). It is apparent that the initial estimate corresponding to the true zone (i.e. the zone where the true platform trajectory is) is near the true platform trajectory in both cases; incidentally a degree of similarity is also present in the estimate corresponding to a different zone in scenario (ii) (zone (b), cf. Fig. 10). Nonetheless, in both cases the procedure produces a very good initial estimate corresponding to the zone where $\breve{x}_{P}^{s}$ belongs, which bodes well for a local optimization routine.

The convergence properties of the algorithm are illustrated in Figs. 7 and 11 for the three different initial estimates, and the performance is analyzed in terms of the time-averaged rootsquare position-error (RSPE) defined as

$$
\operatorname{RSPE}(m) \triangleq \frac{1}{n} \sum_{i=1}^{n}\left\|\hat{\boldsymbol{p}}_{P}^{m}\left(t_{i}\right)-\breve{\boldsymbol{p}}_{P}\left(t_{i}\right)\right\|_{2}
$$

with $\hat{\boldsymbol{p}}_{P}^{m}(\cdot)$ denoting the output of the local optimization routine after $m$ iterations. It is apparent that there is no monotonic decrease of the RSPE, since the maximization of the objection function is conducted w.r.t. the vector $\boldsymbol{x}_{P}^{s}$; however convergence is observed with an acceptable number of iterations (recall that there is no need to compute the Jacobian at each iteration and so each iteration is very light from a computational point of view). In Fig. 8 (resp. Fig. 12) we finally show the platform corresponding to the maximum of the three outputs obtained with the NelderMead method and the three different initial estimates. It is apparent that in both the scenarios

\footnotetext{
${ }^{10}$ It is worth remarking that in such a case $\left[\frac{\alpha_{\theta, \max }-\alpha_{\theta, m i n}}{2 \breve{\alpha}_{\theta}}+1\right] \approx 1.5$, thus $N_{\theta}=5$ for this particular scenario is a fair choice.
} 


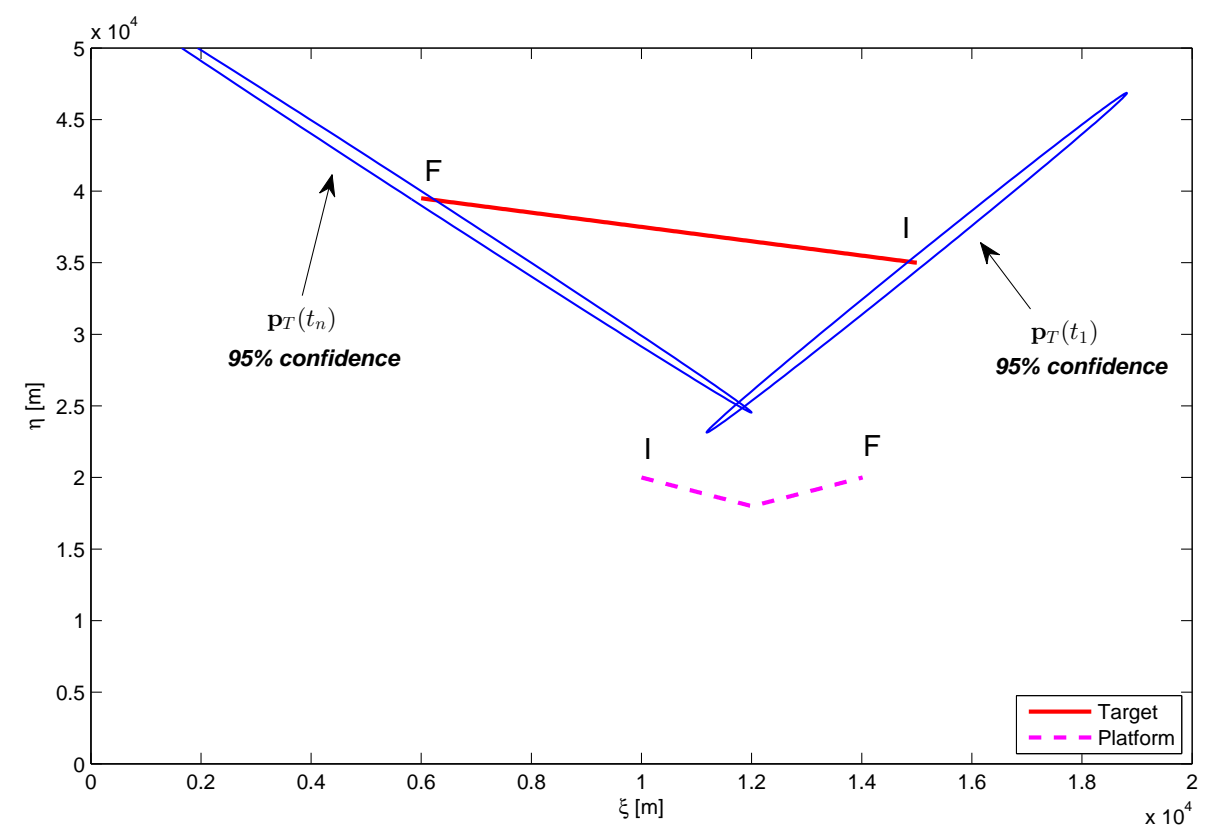

Figure 5: Platform and target trajectories considered for scenario $(i)$.

the true platform trajectory is identified exactly (recall that this is a deterministic problem); such results confirm our conjecture on the uniqueness of the Eq. (23).

\section{Turning time $t_{k}$ sensitivity analysis}

In this paragraph we will remove the assumption that the turning time $t_{k}$ is known at the target-friendly side and we will show the effects of a grid search for the turning time index, denoted as $k$, for both scenarios $(i)$ and (ii). Figs. 13 and 14 show the RSPE (obtained as the minimum along the three zones) as a function of the assumed index $k$. It is apparent that the true platform trajectory is still identifiable in both scenarios; however the RSPE w.r.t. to the index $k$ is not a unimodal (discrete) function and therefore no "naïve" golden-search method can be applied to identify the platform; rather a parallel approach is needed.

Finally it is worth noting that the overall complexity $\Xi$ of the proposed approach is given by:

$$
\begin{aligned}
\Xi & \triangleq \sum_{k=1}^{n} \omega_{a}(k)+\omega_{b}(k)+\omega_{c}(k) \\
& =\bar{\omega} \sum_{k=1}^{n} \chi_{a}(k)+\chi_{b}(k)+\chi_{c}(k) \\
\omega_{i}(k) & \triangleq \bar{\omega} \cdot \chi_{i}(k)
\end{aligned}
$$




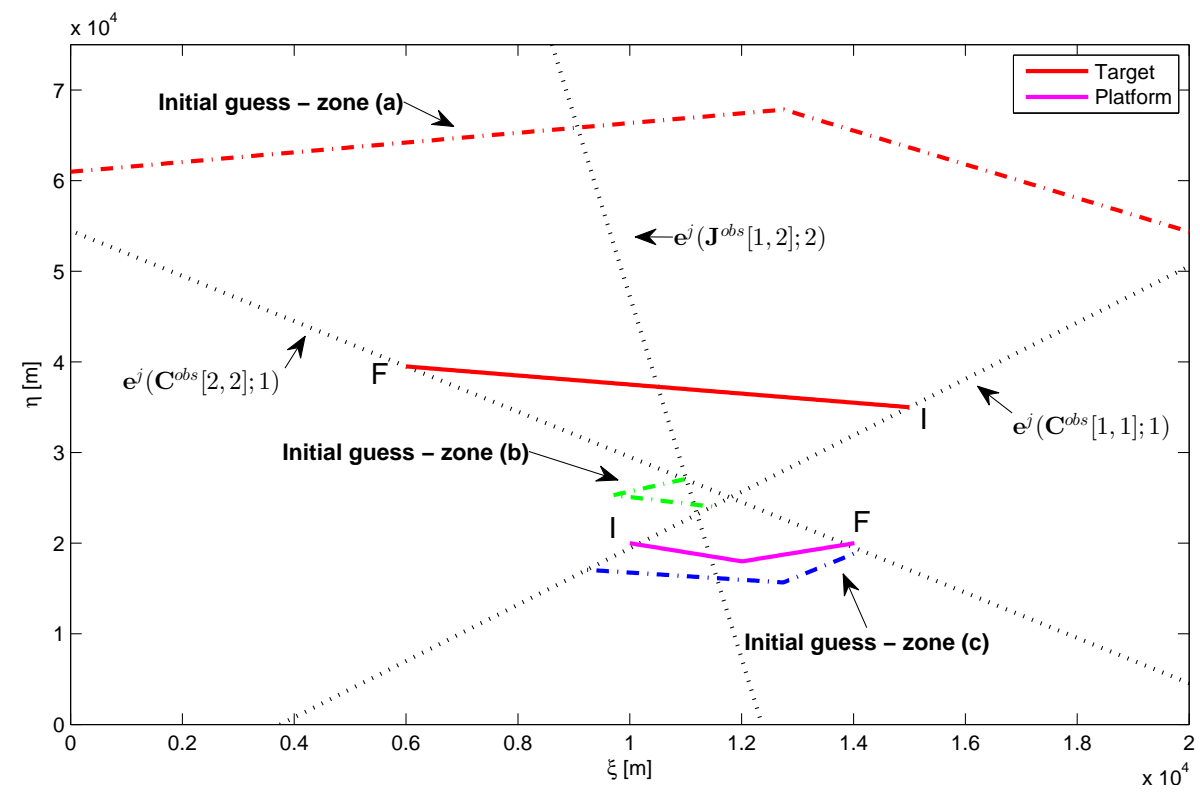

Figure 6: Geometry-driven initial guess procedure for platform trajectory in scenario $(i)$.

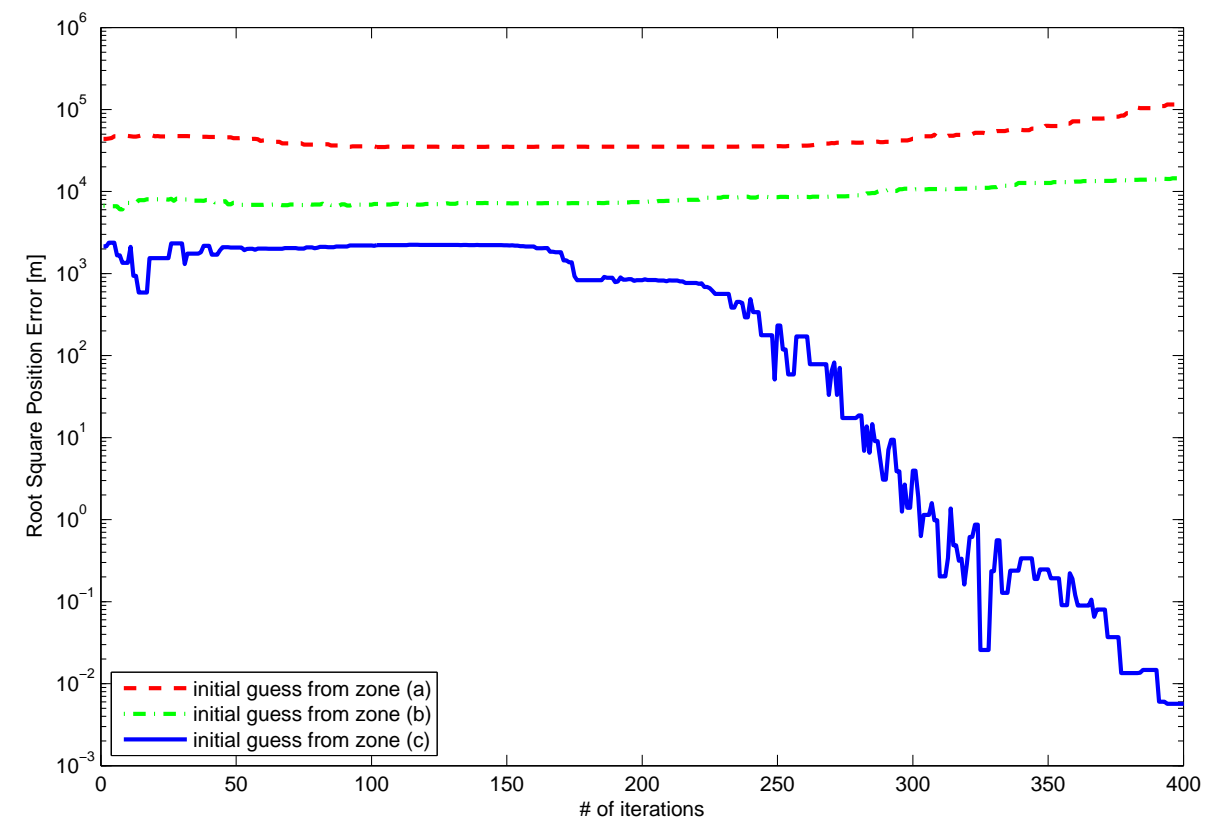

Figure 7: RSPE as a function of the number of iterations for scenario (i). 


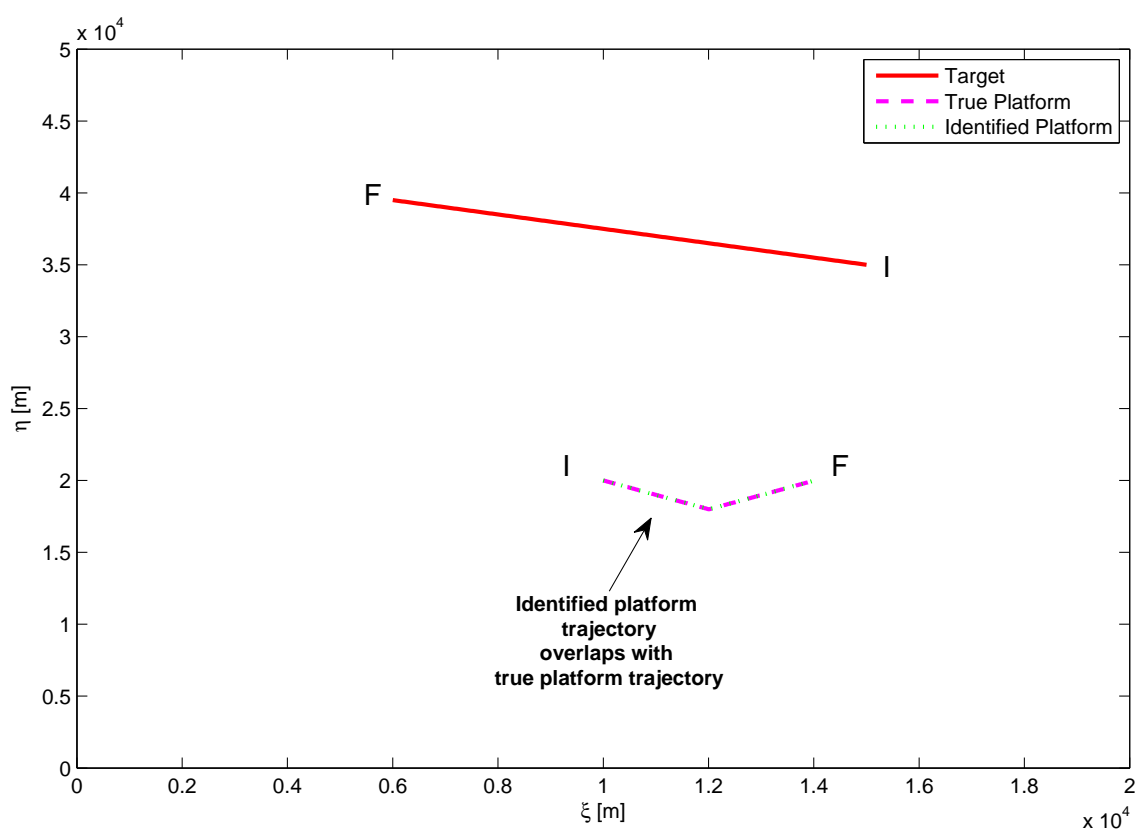

Figure 8: Identification results for scenario $(i)$.

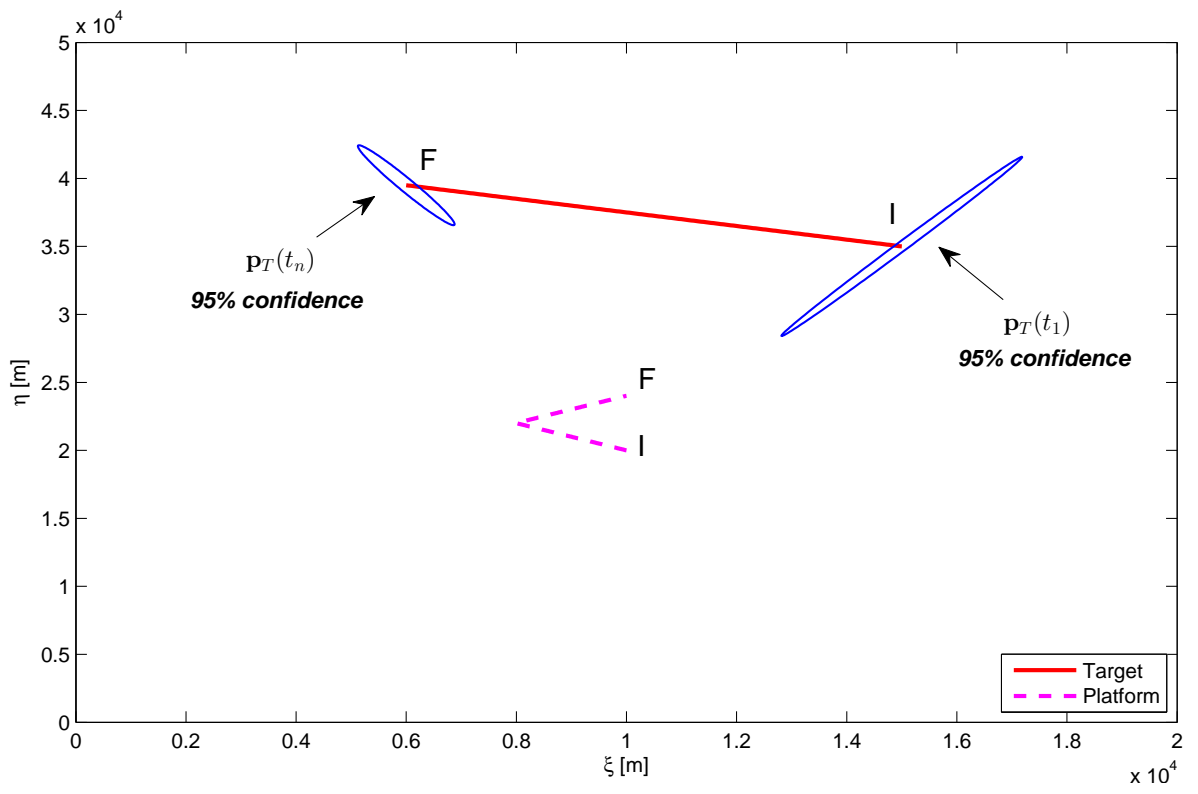

Figure 9: Platform and target trajectories considered for scenario (ii). 


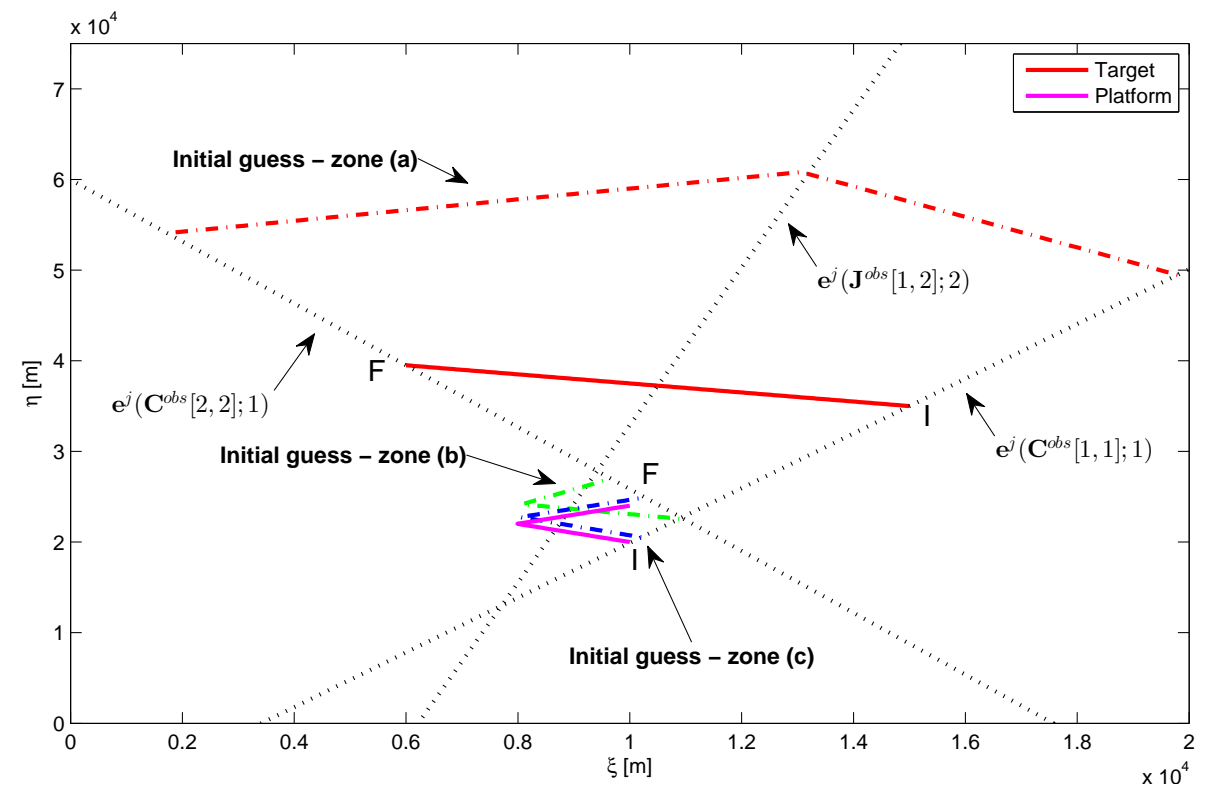

Figure 10: Geometry-driven initial guess procedure for platform trajectory in scenario (ii).

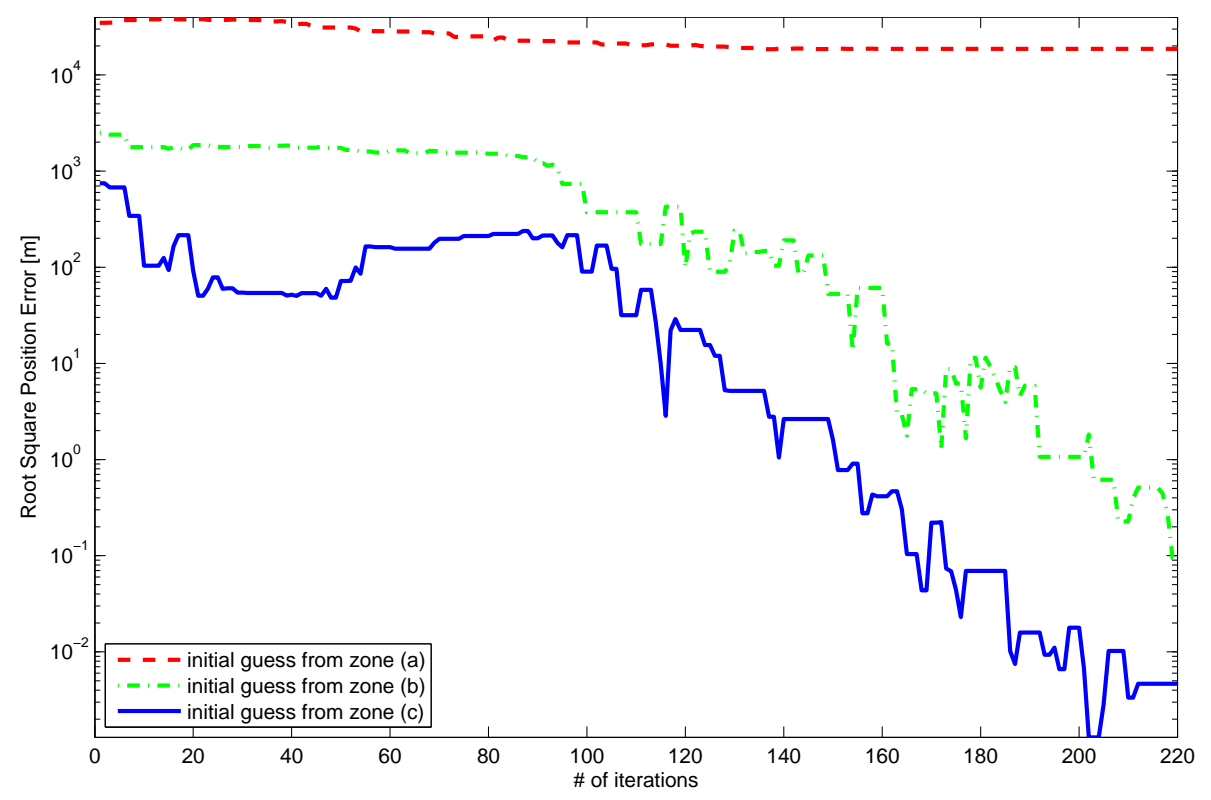

Figure 11: RSPE as a function of the number of iterations for scenario (ii). 


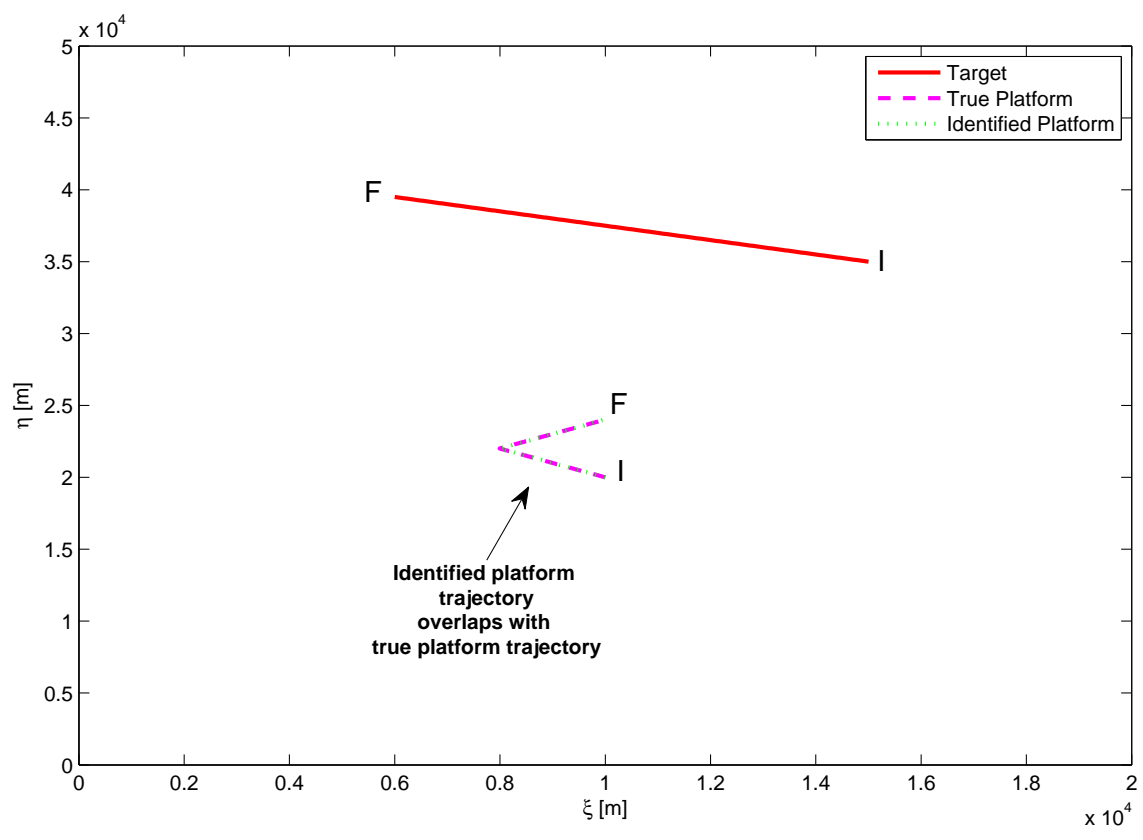

Figure 12: Identification results for scenario $(i i)$.

where $\omega_{i}(k)$ denotes the complexity of the Nelder-Mead method with initial estimate belonging to zone $i$ and assumed index $k$. Note that $\omega_{i}(k)$ is simply given by the complexity of the single iteration $\bar{\omega}$, multiplied by the number of iterations $\chi_{i}(k)$. It is worth noting that there is no convergence theory to support an analysis providing an estimate for the number of iterations required to to satisfy any reasonable accuracy constraint, given as a stopping condition [27]. Instead, regarding the complexity of the single iteration, it has been proved in [27] that $\bar{\omega}$ has a complexity which is only dependent on the dimension of the search space, which is fivedimensional in our case (since $\boldsymbol{x}_{P}^{s} \in \mathbb{R}^{5}$ ).

\section{CONCLUSIONS}

In this paper we studied the problem of identifying the platform motion from its ML-PDA estimation results on an observed target - the estimation of the stealthy estimator. We have addressed only the common "two-leg" platform trajectory (see Figs. 1, 2, 3, 4 etc.); a similar analysis could be performed to other platform trajectories (such as a constant speed turn). We demonstrated that even a general "two-leg" platform motion model can lead to ambiguity in the identification; however, imposition of the constraint of constant speed motion ensures 


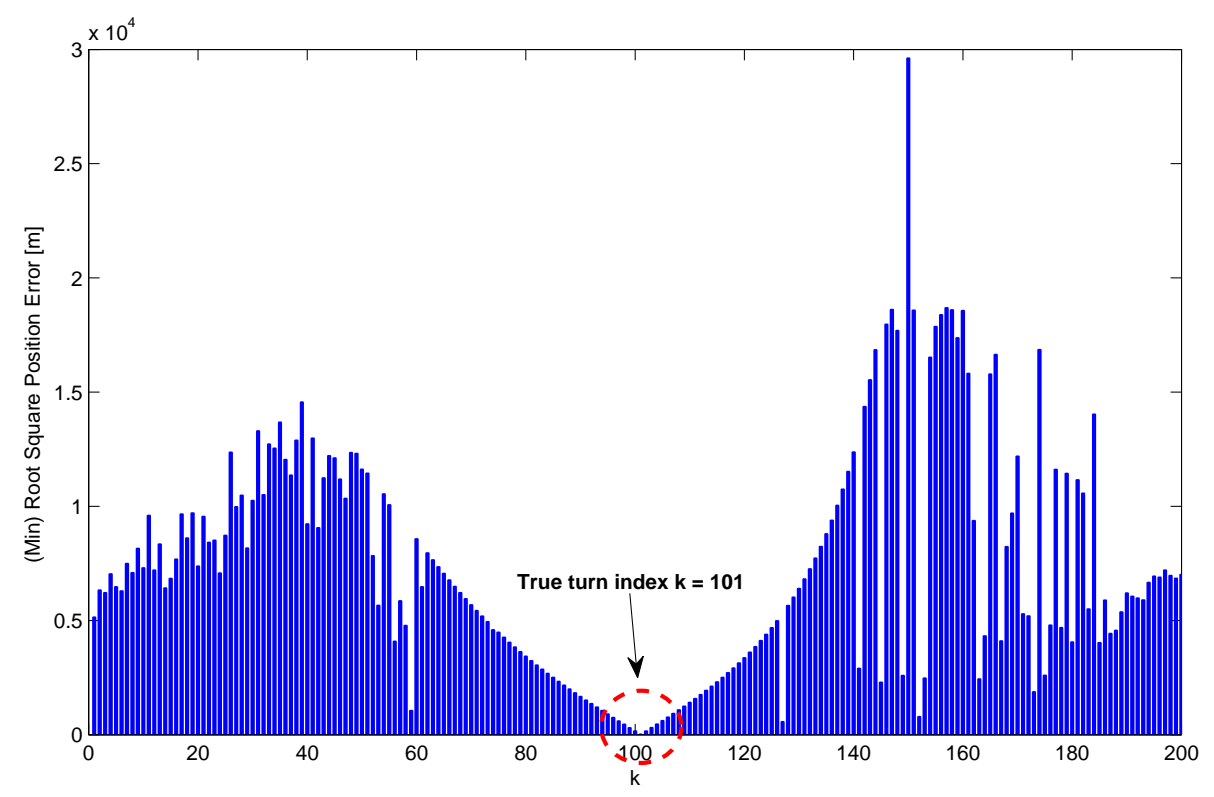

Figure 13: Sensitivity analysis in scenario $(i)$.

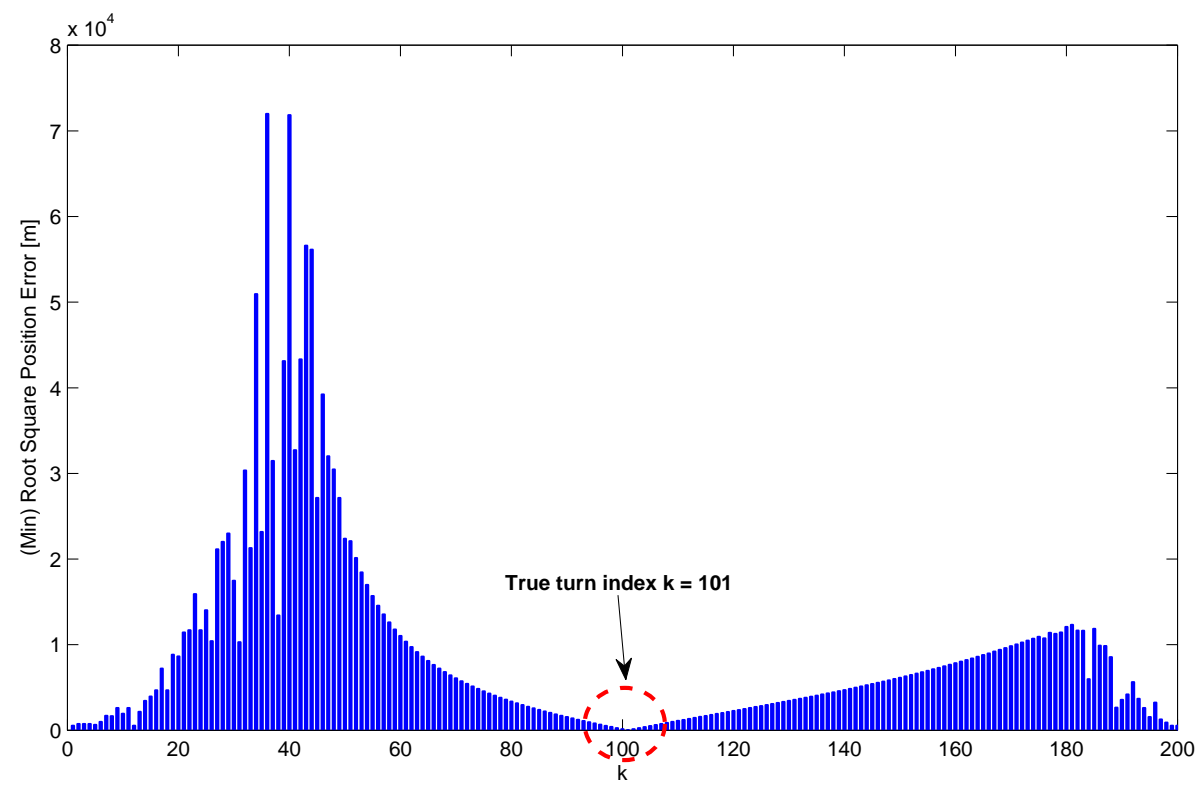

Figure 14: Sensitivity analysis in scenario (ii). 
observability of the platform in most of the scenarios. We modelled the problem as a Frobenius norm minimization and we found a convenient objective function, exploiting the FIM elements and independent on the platform measurement-related parameters, namely $\alpha_{\theta}$. Also, we devised a procedure for the choice of a very small set of initial estimates as input for the numerical optimization procedure, on the basis of theoretical considerations on the geometry of the FIM. Finally, we corroborated the theoretical findings and we have shown the effectiveness of the approach for the choice of the initial estimates, through simulation results. Future research will tackle incomplete (and noisy) intercepted information and different platform motion models. Note that although we have taken the ML-PDA as the underlying algorithm this is only in attempt at generality. In fact, the results presented here apply also to situations with any degree of measurement origin uncertainty, including of course the situation of a deterministic target observed via "clean" measurements without false alarms or missed detections.

\section{ACKNOWLEDGEMENT}

The authors would like to thank the editor and the anonymous reviewers for their valuable comments and especially for one encouraging us to address one particular key issue of observability.

\section{APPENDIX A}

\section{PROOF OF PROPOSITION 1}

To prove this proposition let us assume that there exists a solution $\left\{\boldsymbol{x}_{P}^{*}, \alpha_{\theta}^{*}\right\}$ such that $\boldsymbol{J}\left(\hat{\boldsymbol{x}}_{T}, \boldsymbol{x}_{P}^{*}, \alpha_{\theta}^{*}\right)=$ $\boldsymbol{J}^{\text {obs }}$. Now let us consider the subspace $\left\{\boldsymbol{x}_{P}^{\prime}, \alpha_{\theta}^{\prime}\right\}$ defined as

$$
\left\{\boldsymbol{x}_{P}^{\prime}, \alpha_{\theta}^{\prime}\right\}=\left\{\beta \boldsymbol{x}_{P}^{*}+(1-\beta) \overline{\boldsymbol{x}}_{E}, \beta^{2} \alpha_{\theta}^{*}\right\}, \quad \beta \in \mathbb{R}
$$

where $\overline{\boldsymbol{x}}_{E} \triangleq\left[\begin{array}{ccc}\hat{\boldsymbol{p}}_{T}\left(t_{1}\right)^{t} & \hat{\boldsymbol{v}}_{T}^{t} & \hat{\boldsymbol{v}}_{T}^{t}\end{array}\right]^{t}$. The subspace contains the set of platform trajectories whose velocity and position vectors are linear combinations of the ones of the platform trajectory defined by $\boldsymbol{x}_{P}^{*}$ and the estimated target trajectory.

In this case the corresponding $\theta_{i}\left(\hat{\boldsymbol{x}}_{T}, \boldsymbol{x}_{P}^{\prime}\right)$ and $r_{i}\left(\hat{\boldsymbol{x}}_{T}, \boldsymbol{x}_{P}^{\prime}\right)$ have the explicit expressions

$$
\begin{aligned}
\theta_{i}\left(\hat{\boldsymbol{x}}_{T}, \boldsymbol{x}_{P}^{\prime}\right) & =\arctan \left(\frac{\hat{\xi}_{T}\left(t_{i}\right)-\left(\beta \xi_{P}\left(t_{i}\right)+(1-\beta) \hat{\xi}_{T}\left(t_{i}\right)\right)}{\hat{\eta}_{T}\left(t_{i}\right)-\left(\beta \eta_{P}\left(t_{i}\right)+(1-\beta) \hat{\eta}_{T}\left(t_{i}\right)\right)}\right) \\
& =\theta_{i}\left(\hat{\boldsymbol{x}}_{T}, \boldsymbol{x}_{P}^{*}\right)
\end{aligned}
$$




$$
\begin{aligned}
r_{i}\left(\hat{\boldsymbol{x}}_{T}, \boldsymbol{x}_{P}^{\prime}\right) & =\sqrt{\left(\hat{\xi}_{T}\left(t_{i}\right)-\left(\beta \xi_{P}\left(t_{i}\right)+(1-\beta) \hat{\xi}_{T}\left(t_{i}\right)\right)\right)^{2}+\left(\hat{\eta}_{T}\left(t_{i}\right)-\left(\beta \eta_{P}\left(t_{i}\right)+(1-\beta) \hat{\eta}_{T}\left(t_{i}\right)\right)\right)^{2}} \\
& =\beta r_{i}\left(\hat{\boldsymbol{x}}_{T}, \boldsymbol{x}_{P}^{*}\right)
\end{aligned}
$$

By plugging Eqs. (56) and (57) into Eq. (12) we obtain the equality

$$
\boldsymbol{\nabla}_{\boldsymbol{x}_{T}}\left(\theta_{i}\left(\hat{\boldsymbol{x}}_{T}, \boldsymbol{x}_{P}^{\prime}\right)\right)=\frac{1}{\beta} \boldsymbol{\nabla}_{\boldsymbol{x}_{T}}\left(\theta_{i}\left(\hat{\boldsymbol{x}}_{T}, \boldsymbol{x}_{P}^{*}\right)\right)
$$

Using Eq. (58) we can express $\boldsymbol{J}\left(\hat{\boldsymbol{x}}_{T}, \boldsymbol{x}_{P}^{\prime}, \alpha_{\theta}^{\prime}\right)$ as

$$
\begin{aligned}
\boldsymbol{J}\left(\hat{\boldsymbol{x}}_{T}, \boldsymbol{x}_{P}^{\prime}, \alpha_{\theta}^{\prime}\right) & =\left(\beta^{2} \alpha_{\theta}^{*}\right) \sum_{i=0}^{n} \frac{1}{\beta} \boldsymbol{\nabla}_{\boldsymbol{x}_{T}}\left(\theta_{i}\left(\hat{\boldsymbol{x}}_{T}, \boldsymbol{x}_{P}^{*}\right)\right) \frac{1}{\beta} \boldsymbol{\nabla}_{\boldsymbol{x}_{T}}^{t}\left(\theta_{i}\left(\hat{\boldsymbol{x}}_{T}, \boldsymbol{x}_{P}^{*}\right)\right) \\
& =\boldsymbol{J}\left(\hat{\boldsymbol{x}}_{T}, \boldsymbol{x}_{P}^{*}, \alpha_{\theta}^{*}\right)
\end{aligned}
$$

Since $\left\{\boldsymbol{x}_{P}^{*}, \alpha_{\theta}^{*}\right\}$ and $\left\{\boldsymbol{x}_{P}^{\prime}, \alpha_{\theta}^{\prime}\right\}$ (independently of $\beta$ ) lead both to $\boldsymbol{J}^{o b s}, \boldsymbol{J}(\cdot)$ does not represent a one-to-one mapping, which makes the platform-state vector $\boldsymbol{x}_{P}$ unidentifiable.

\section{APPENDIX B}

\section{PROOF OF LEMMA 2}

We start by observing that $\boldsymbol{J}\left(\hat{\boldsymbol{x}}_{T}, \boldsymbol{x}_{P}^{s}, \alpha_{\theta}\right)$ and $\boldsymbol{\nabla}_{\boldsymbol{x}_{T}}\left(\theta_{i}\left(\hat{\boldsymbol{x}}_{T}, \boldsymbol{x}_{P}^{s}\right)\right)$ can be expressed similarly as Eqs. (9) and (12). Also, for notational convenience let us define

$$
\boldsymbol{y}_{i} \triangleq \frac{1}{r_{i}\left(\hat{\boldsymbol{x}}_{T}, \boldsymbol{x}_{P}^{s}\right)}\left[\cos \left(\theta_{i}\left(\hat{\boldsymbol{x}}_{T}, \boldsymbol{x}_{P}^{s}\right)\right)-\sin \left(\theta_{i}\left(\hat{\boldsymbol{x}}_{T}, \boldsymbol{x}_{P}^{s}\right)\right)\right]^{t}
$$

and rewrite $\boldsymbol{\nabla}_{\boldsymbol{x}_{T}}\left(\theta_{i}\left(\hat{\boldsymbol{x}}_{T}, \boldsymbol{x}_{P}^{s}\right)\right)$ as

$$
\boldsymbol{\nabla}_{\boldsymbol{x}_{T}}\left(\theta_{i}\left(\boldsymbol{x}_{T}, \boldsymbol{x}_{P}^{s}\right)\right)=\left[\begin{array}{c}
\left(1-\alpha_{i}\right) \boldsymbol{y}_{i} \\
\alpha_{i} \boldsymbol{y}_{i}
\end{array}\right]
$$

where $\alpha_{i}$ has been defined in Eq. (2). Substituting Eq. (62) into the explicit form of $\boldsymbol{J}\left(\hat{\boldsymbol{x}}_{T}, \boldsymbol{x}_{P}^{s}, \alpha_{\theta}\right)$, we get

$$
\begin{aligned}
\boldsymbol{J}\left(\hat{\boldsymbol{x}}_{T}, \boldsymbol{x}_{P}^{s}, \alpha_{\theta}\right) \triangleq\left[\begin{array}{cc}
\boldsymbol{J}[1,1] & \boldsymbol{J}[1,2] \\
\boldsymbol{J}[2,1] & \boldsymbol{J}[2,2]
\end{array}\right] \\
=\alpha_{\theta}\left[\begin{array}{cc}
\sum_{i=1}^{n}\left(1-\alpha_{i}\right)^{2} \boldsymbol{y}_{i} \boldsymbol{y}_{i}^{t} & \sum_{i=1}^{n} \alpha_{i}\left(1-\alpha_{i}\right) \boldsymbol{y}_{i} \boldsymbol{y}_{i}^{t} \\
\sum_{i=1}^{n} \alpha_{i}\left(1-\alpha_{i}\right) \boldsymbol{y}_{i} \boldsymbol{y}_{i}^{t} & \sum_{i=1}^{n} \alpha_{i}{ }^{2} \boldsymbol{y}_{i} \boldsymbol{y}_{i}^{t}
\end{array}\right]
\end{aligned}
$$

where we have elucidated the block-decomposition arising from Eq. (62) into [2 $\times 2]$ matrices $\boldsymbol{J}[\ell, m], \ell, m \in\{1,2\}$. Since we have that $\boldsymbol{J}[1,2]=\boldsymbol{J}[2,1]$, only three matrices contain nonrepeated entries (i.e., the 4 entries of $\boldsymbol{J}[1,2]$ or $\boldsymbol{J}[2,1]$ can be neglected). Also, since $\boldsymbol{J}[1,1]$, 
$\boldsymbol{J}[2,1]$ and $\boldsymbol{J}[2,2]$ are symmetric matrices, there is a repeated entry in each of them, thus leading to 3 other dependent entries. Therefore the 16 entries of the FIM actually contain only 9 independent elements. W.l.o.g. we consider here (and throughout the paper), the following independent entries (we drop the dependence w.r.t. $\hat{\boldsymbol{x}}_{T}, \boldsymbol{x}_{P}^{s}$ and $\alpha_{\theta}$ ) and we denote with $\mathcal{C}$ the corresponding set of indices:

$$
\left\{J_{1,1}, J_{2,2}, J_{3,3}, J_{4,4}, J_{1,2}, J_{1,3}, J_{1,4}, J_{2,4}, J_{3,4}\right\}
$$

\section{APPENDIX C}

\section{Proof of Proposition 3}

We have shown, through Lemma 2 , that $\boldsymbol{J}\left(\boldsymbol{x}_{P}^{s}, \alpha_{\theta}\right)$ has only 9 independent entries. Also, the dependent entries are simple repetitions of the corresponding independent entries, due to particular symmetry structure of the FIM (cfr. Eq. (63)).

The same argument extends to $\boldsymbol{D}\left(\boldsymbol{x}_{P}^{s}, \alpha_{\theta}\right) \triangleq\left(\boldsymbol{J}^{\text {obs }}-\boldsymbol{J}\left(\boldsymbol{x}_{P}^{s}, \alpha_{\theta}\right)\right)$ and its entry-wise squared version $\overline{\boldsymbol{D}}\left(\boldsymbol{x}_{P}^{s}, \alpha_{\theta}\right)$ (i.e. $\left.\bar{D}_{\ell, m}\left(\boldsymbol{x}_{P}^{s}, \alpha_{\theta}\right)=D_{\ell, m}^{2}\left(\boldsymbol{x}_{P}^{s}, \alpha_{\theta}\right)\right)$, iff $\boldsymbol{J}^{\text {obs }}$ retains the same property of symmetry; this is accomplished if $\boldsymbol{J}^{\text {obs }}$ is a noise-free observed FIM, that is $\boldsymbol{J}^{o b s}=\boldsymbol{J}\left(\breve{\boldsymbol{x}}_{P}^{s}, \breve{\alpha}_{\theta}\right)$.

By construction, the following equality holds

$$
\left\|\boldsymbol{J}^{o b s}-\boldsymbol{J}\left(\boldsymbol{x}_{P}^{s}, \alpha_{\theta}\right)\right\|_{F}^{2}=\sum_{\ell=1}^{4} \sum_{m=1}^{4} \bar{D}_{\ell, m}\left(\boldsymbol{x}_{P}^{s}, \alpha_{\theta}\right)
$$

This sum can be efficiently evaluated by considering only $(\ell, m) \in \mathcal{C}$, with $\mathcal{C}$ being the set of the independent entries according to Eq. (65), and weighting them by the number of times they are repeated in the matrix $\bar{D}$. Thus the 1.h.s. of Eq. (66) can be rewritten as

$$
\sum \sum_{(\ell, m) \in \mathcal{C}} W_{\ell, m} \bar{D}_{\ell, m}\left(\boldsymbol{x}_{P}^{s}, \alpha_{\theta}\right)=\sum \sum_{(\ell, m) \in \mathcal{C}} W_{\ell, m}\left(J_{\ell, m}^{o b s}-J_{\ell, m}\left(\boldsymbol{x}_{P}^{s}, \alpha_{\theta}\right)\right)^{2}
$$

By stacking the elements $J_{\ell, m}^{o b s}$ (resp. $J_{\ell, m}\left(\boldsymbol{x}_{P}^{s}, \alpha_{\theta}\right)$ ), $(\ell, m) \in \mathcal{C}$, into the vector $\boldsymbol{j}^{\text {obs }}$ (resp. $\boldsymbol{j}\left(\boldsymbol{x}_{P}^{s}, \alpha_{\theta}\right)$ ), and defining the diagonal matrix $\boldsymbol{W}$ with elements $W_{\ell, m}$, we obtain the weighted form of Eq. (25). Finally, it is straightforward to show that $W$ has the expression

$$
\boldsymbol{W}=\operatorname{diag}\left(\left[\begin{array}{lllllllll}
1 & 1 & 1 & 1 & 2 & 2 & 4 & 2 & 2
\end{array}\right]^{t}\right)
$$

where the weight equal to 4 accounts for the repeated elements along the minor (secondary) diagonal, while the weights equal to 2 account for the remaining symmetric entries into each block matrix $\boldsymbol{J}[\ell, m], \ell, m \in\{1,2\}$, and between the matrices $\boldsymbol{J}[1,2]$ and $\boldsymbol{J}[2,1]$. 


\section{APPENDIX D}

\section{ProOF OF PROPOSITION 4}

Let us rewrite Eq. (27) here for convenience:

$$
\mathcal{F}\left(\boldsymbol{x}_{P}^{s}, \alpha_{\theta}\right)=\left[\boldsymbol{j}^{o b s}-\boldsymbol{j}_{u}\left(\boldsymbol{x}_{P}^{s}\right) \alpha_{\theta}\right]^{t} \boldsymbol{W}\left[\boldsymbol{j}^{o b s}-\boldsymbol{j}_{u}\left(\boldsymbol{x}_{P}^{s}\right) \alpha_{\theta}\right]
$$

For a fixed $\boldsymbol{x}_{P}^{s}$ it is easy to check that the observation model in Eq. (69) is linear in $\alpha_{\theta}$. Therefore given $\boldsymbol{x}_{P}^{s}, \hat{\alpha}_{\theta}$ can be found as the solution of a standard least squares problem [20] as

$$
\hat{\alpha}_{\theta}=\frac{\boldsymbol{j}_{u}\left(\boldsymbol{x}_{P}^{s}\right)^{t} \boldsymbol{W} \boldsymbol{j}^{o b s}}{\boldsymbol{j}_{u}\left(\boldsymbol{x}_{P}^{s}\right)^{t} \boldsymbol{W} \boldsymbol{j}_{u}\left(\boldsymbol{x}_{P}^{s}\right)}
$$

Then substituting Eq. (70) in Eq. (69) we obtain the expression for $\mathcal{F}\left(\boldsymbol{x}_{P}^{s}, \alpha_{\theta}\right)$

$$
\mathcal{F}\left(\boldsymbol{x}_{P}^{s}, \hat{\alpha}_{\theta}\right)=\left[\boldsymbol{j}^{o b s}-\boldsymbol{j}_{u}\left(\boldsymbol{x}_{P}^{s}\right) \frac{\boldsymbol{j}_{u}\left(\boldsymbol{x}_{P}^{s}\right)^{t} \boldsymbol{W} \boldsymbol{j}^{o b s}}{\boldsymbol{j}_{u}\left(\boldsymbol{x}_{P}^{s}\right)^{t} \boldsymbol{W} \boldsymbol{j}_{u}\left(\boldsymbol{x}_{P}^{s}\right)}\right]^{t} \boldsymbol{W}\left[\boldsymbol{j}^{o b s}-\boldsymbol{j}_{u}\left(\boldsymbol{x}_{P}^{s}\right) \frac{\boldsymbol{j}_{u}\left(\boldsymbol{x}_{P}^{s}\right)^{t} \boldsymbol{W} \boldsymbol{j}^{o b s}}{\boldsymbol{j}_{u}\left(\boldsymbol{x}_{P}^{s}\right)^{t} \boldsymbol{W} \boldsymbol{j}_{u}\left(\boldsymbol{x}_{P}^{s}\right)}\right]
$$

Exploiting the orthogonality principle of linear least squares [20], Eq. (71) reduces to

$$
\mathcal{F}\left(\boldsymbol{x}_{P}^{s}, \hat{\alpha}_{\theta}\right)=\left(\boldsymbol{j}^{o b s}\right)^{t}\left(\boldsymbol{W}-\frac{\boldsymbol{W} \boldsymbol{j}_{u}\left(\boldsymbol{x}_{P}^{s}\right) \boldsymbol{j}_{u}\left(\boldsymbol{x}_{P}^{s}\right)^{t} \boldsymbol{W}}{\boldsymbol{j}_{u}\left(\boldsymbol{x}_{P}^{s}\right)^{t} \boldsymbol{W} \boldsymbol{j}_{u}\left(\boldsymbol{x}_{P}^{s}\right)}\right) \boldsymbol{j}^{o b s}
$$

Since $\left(\boldsymbol{j}^{o b s}\right)^{t} \boldsymbol{W} \boldsymbol{j}^{\text {obs }}$ in Eq. (72) does not depend on $\boldsymbol{x}_{P}^{s}$, it is irrelevant in the minimization. Thus neglecting it and considering the opposite of the remaining term, we can equivalently maximize:

$$
\mathcal{G}\left(\boldsymbol{x}_{P}^{s}\right) \triangleq\left(\boldsymbol{j}^{o b s}\right)^{t} \frac{\boldsymbol{W} \boldsymbol{j}_{u}\left(\boldsymbol{x}_{P}^{s}\right) \boldsymbol{j}_{u}\left(\boldsymbol{x}_{P}^{s}\right)^{t} \boldsymbol{W}}{\boldsymbol{j}_{u}\left(\boldsymbol{x}_{P}^{s}\right)^{t} \boldsymbol{W} \boldsymbol{j}_{u}\left(\boldsymbol{x}_{P}^{s}\right)} \boldsymbol{j}^{\text {obs }}
$$

Finally, by defining $\boldsymbol{c}\left(\boldsymbol{x}_{P}^{s}\right) \triangleq \frac{\boldsymbol{W} \boldsymbol{j}_{u}\left(\boldsymbol{x}_{P}^{s}\right)}{\left[\boldsymbol{j}_{u}\left(\boldsymbol{x}_{P}^{s}\right)^{t} \boldsymbol{W} \boldsymbol{j}_{u}\left(\boldsymbol{x}_{P}^{s}\right)\right]^{1 / 2}}$, we can rewrite Eq. (73) as

$$
\begin{aligned}
\mathcal{G}\left(\boldsymbol{x}_{P}^{s}\right) & =\left(\boldsymbol{j}^{o b s}\right)^{t} \boldsymbol{c}\left(\boldsymbol{x}_{P}^{s}\right) \boldsymbol{c}\left(\boldsymbol{x}_{P}^{s}\right)^{t} \boldsymbol{j}^{o b s} \\
& =\left\langle\boldsymbol{j}^{o b s}, \boldsymbol{c}\left(\boldsymbol{x}_{P}^{s}\right)\right\rangle^{2}
\end{aligned}
$$

which concludes the proof.

\section{APPENDIX E}

\section{CHOICE OF $\left\{\hat{r}_{1}, \hat{r}_{n}\right\}$}

In this section we will derive the expressions in Eq. (33). For the sake of simplicity we will use the short-hand notations $r_{i} \triangleq r_{i}\left(\hat{\boldsymbol{x}}_{T}, \breve{\boldsymbol{x}}_{P}^{s}\right)$ and $\theta_{i} \triangleq \theta_{i}\left(\hat{\boldsymbol{x}}_{T}, \breve{\boldsymbol{x}}_{P}^{s}\right)$. We start by considering 
the block decomposition of $\boldsymbol{J}^{\text {obs }}$, through Eq. (63). Let us focus in particular on $\boldsymbol{J}^{\text {obs }}[1,1]$ and $\boldsymbol{J}^{\text {obs }}[2,2]$, i.e. the diagonal blocks, whose explicit expressions are given by

$$
\begin{aligned}
& \boldsymbol{J}^{o b s}[1,1]=\breve{\alpha}_{\theta} \sum_{i=1}^{n} \frac{\left(1-\alpha_{i}\right)^{2}}{r_{i}^{2}} \underbrace{\left[\begin{array}{cc}
\cos ^{2}\left(\theta_{i}\right) & -1 / 2 \sin \left(2 \theta_{i}\right) \\
-1 / 2 \sin \left(2 \theta_{i}\right) & \sin ^{2}\left(\theta_{i}\right)
\end{array}\right]}_{\triangleq \boldsymbol{D}_{i}} \\
& \boldsymbol{J}^{o b s}[2,2]=\breve{\alpha}_{\theta} \sum_{i=1}^{n} \frac{\alpha_{i}{ }^{2}}{r_{i}^{2}} \underbrace{\left[\begin{array}{cc}
\cos ^{2}\left(\theta_{i}\right) & -1 / 2 \sin \left(2 \theta_{i}\right) \\
-1 / 2 \sin \left(2 \theta_{i}\right) & \sin ^{2}\left(\theta_{i}\right)
\end{array}\right]}_{\triangleq \boldsymbol{D}_{i}}
\end{aligned}
$$

note that an analogous expression holds for $\boldsymbol{J}^{\text {obs }}[1,2]$, i.e. $\boldsymbol{J}^{\text {obs }}[1,2]=\breve{\alpha}_{\theta} \sum_{i=1}^{n} \frac{\alpha_{i}\left(1-\alpha_{i}\right)}{r_{i}^{2}} \boldsymbol{D}_{i}$. It can be readily shown, through Eqs. (76) and (77), that $\operatorname{tr}\left(\boldsymbol{J}^{\text {obs }}[\ell, \ell]\right), \ell \in\{1,2\}$, are given by

$$
\begin{aligned}
& \operatorname{tr}\left(\boldsymbol{J}^{o b s}[1,1]\right)=\breve{\alpha}_{\theta}\left[\frac{1}{r_{1}^{2}}+\sum_{i=2}^{n} \frac{\left(1-\alpha_{i}\right)^{2}}{r_{i}^{2}}\right] \\
& \operatorname{tr}\left(\boldsymbol{J}^{o b s}[2,2]\right)=\breve{\alpha}_{\theta}\left[\frac{1}{r_{n}^{2}}+\sum_{i=1}^{n-1} \frac{\alpha_{i}^{2}}{r_{i}^{2}}\right]
\end{aligned}
$$

From inspection of Eqs. (78) and (79), it is apparent that each trace is a weighted sum (scaled by $\breve{\alpha}_{\theta}$ ) of $\frac{1}{r_{i}^{2}}$. Note that, by definition, the following inequalities hold $\forall i \in \mathcal{I}$ :

$$
\left(1-\alpha_{i}\right)^{2}<\left(1-\alpha_{i+1}\right)^{2}, \quad \alpha_{i+1}{ }^{2}<\alpha_{i}^{2}
$$

By exploiting them, we have that in $\operatorname{tr}\left(\boldsymbol{J}^{\text {obs }}[1,1]\right)\left(\right.$ resp. $\left.\operatorname{tr}\left(\boldsymbol{J}^{\text {obs }}[2,2]\right)\right)$ the term $\frac{1}{r_{1}^{2}}\left(\right.$ resp. $\left.\frac{1}{r_{n}^{2}}\right)$ receives the highest weight, while the term $\frac{1}{r_{n}^{2}}$ (resp. $\frac{1}{r_{1}^{2}}$ ) contributes to the sum with zero weight. To obtain a good approximation (and avoid biased estimates) of $\left\{r_{1}, r_{n}\right\}$ we first consider convex combination counterparts of Eqs. (78) and (79), since the positive weights in $\operatorname{tr}\left(\boldsymbol{J}^{\text {obs }}[1,1]\right)$ and $\operatorname{tr}\left(\boldsymbol{J}^{\text {obs }}[2,2]\right)$ do not satisfy the normalization property (i.e. $\sum_{i=1}^{n}\left(1-\alpha_{i}\right)^{2} \neq 1$ and $\sum_{i=1}^{n} \alpha_{i}{ }^{2} \neq$ 1). The reasons are twofold: $(i)$ the weights $\alpha_{i}$ and $\left(1-\alpha_{i}\right)$ appear in squared form in Eqs. (78) and (79); and (ii) even $\alpha_{i}$ and $\left(1-\alpha_{i}\right)$ do not sum to one ${ }^{11}$. For such a reason we normalize $\operatorname{tr}\left(\boldsymbol{J}^{\text {obs }}[1,1]\right)$ (resp. $\left.\operatorname{tr}\left(\boldsymbol{J}^{\text {obs }}[1,1]\right)\right)$ by $\sum_{i=1}^{n}\left(1-\alpha_{i}\right)^{2}$ (resp. $\left.\sum_{i=1}^{n} \alpha_{i}^{2}\right)$. Then, we note that if

$$
\begin{aligned}
& \text { (a) } r_{i} \approx r_{1}, \quad i \ni \alpha_{i}^{2} \ll 1 \\
& \text { (b) } r_{j} \approx r_{n}, \quad j \ni\left(1-\alpha_{j}\right)^{2} \ll 1
\end{aligned}
$$

\footnotetext{
${ }^{11}$ For example in the case of uniform sampling it holds $\sum_{i=1}^{n} \alpha_{i}=\sum_{i=1}^{n}\left(1-\alpha_{i}\right)=\frac{n}{2}$; thus sum grows proportionally with the sample rate. The growth of $\sum_{i=1}^{n} \alpha_{i}$ and $\sum_{i=1}^{n}\left(1-\alpha_{i}\right)$ with the sample rate is present also under the more general non-uniform sampling assumption, but in the latter case $\sum_{i=1}^{n} \alpha_{i} \neq \sum_{i=1}^{n}\left(1-\alpha_{i}\right) \neq \frac{n}{2}$.
} 
the term $\frac{\operatorname{tr}\left(\boldsymbol{J}^{\text {obs }}[1,1]\right)}{\sum_{i=1}^{n}\left(1-\alpha_{i}\right)^{2}}$ (resp. $\left.\frac{\operatorname{tr}\left(\boldsymbol{J}^{o b s}[2,2]\right)}{\sum_{i=1}^{n} \alpha_{i}{ }^{2}}\right)$ well approximates $\frac{\breve{\alpha}_{\theta}}{r_{1}^{2}}$ (resp. $\frac{\breve{\alpha}_{\theta}}{r_{n}^{2}}$ ), thus leading to Eq. (33); therefore the value of $\operatorname{tr}\left(\boldsymbol{J}^{o b s}[1,1]\right)$ (resp. $\left.\operatorname{tr}\left(\boldsymbol{J}^{o b s}[2,2]\right)\right)$ will be high when the platform trajectory is near to the target at the beginning (resp. at the end) of the observation time. The conditions in Eq. (81) reflect the assumption that ranges at early (resp. late) time samples have non-negligible weights in Eq. (78) (resp. Eq. (79)) but they are very similar to $r_{1}$ (resp. $r_{n}$ ), which is a reasonable assumption in sonar tracking. Also, note that the mentioned condition is weaker than $r_{i} \approx r, \forall i \in \mathcal{I}$, (i.e. an approximately constant range assumption) and includes it as a more restrictive case.

\section{APPENDIX F}

CHOICE OF $\left\{\hat{\boldsymbol{i}}_{1}, \hat{\boldsymbol{i}}_{n}\right\}$

The purpose of this Appendix is to show that: $(i) \boldsymbol{J}^{\text {obs }}$ contains only incomplete information about $\left\{\boldsymbol{i}_{1}, \boldsymbol{i}_{n}\right\}$, meaning that it is not possible to extract these vectors without ambiguity; (ii) a good approach to extract such information is represented by Eq. (37).

For this purpose, let us consider the definition of $\boldsymbol{D}_{i}$ in Eqs. (76) and (77). It can be readily shown that each $\boldsymbol{D}_{i}$ has eigenvalues $\left\{\lambda_{1, i}, \lambda_{2, i}\right\}=\{1,0\}$ and that the corresponding (orthogonal) eigenvectors are

$$
\boldsymbol{e}^{a}\left(\boldsymbol{D}_{i} ; 1\right)=a\left[\begin{array}{c}
\cos \left(\theta_{i}\right) \\
-\sin \left(\theta_{i}\right)
\end{array}\right], \quad \boldsymbol{e}^{a}\left(\boldsymbol{D}_{i} ; 2\right)=a\left[\begin{array}{c}
\sin \left(\theta_{i}\right) \\
\cos \left(\theta_{i}\right)
\end{array}\right], \quad a \in\{-1,1\}
$$

The pair $\left\{\lambda_{1, i}, \boldsymbol{e}^{a}\left(\boldsymbol{D}_{i} ; 1\right)\right\}$ corresponds to the cross-range direction at $t_{i}$, while $\left\{\lambda_{2, i}, \boldsymbol{e}^{a}\left(\boldsymbol{D}_{i} ; 2\right)\right\}$ corresponds to the range direction at $t_{i}$. In fact, recall that each $\boldsymbol{D}_{i}$ has an informative contribution along the cross-range direction, thus $\lambda_{1, i}=1^{12}$; on the other hand each $\boldsymbol{D}_{i}$ has no informative contribution along the range direction (since the range is estimated with at least two bearing measurements), that is $\lambda_{2, i}=0$. For this reason the information related to $\boldsymbol{i}_{1}$ (resp. $\boldsymbol{i}_{n}$ ) is contained in $\boldsymbol{e}^{a}\left(\boldsymbol{D}_{1} ; 2\right)$ (resp. $\left.\boldsymbol{e}^{a}\left(\boldsymbol{D}_{n} ; 2\right)\right)$. Note that however the sign-ambiguity in the definition of $\boldsymbol{e}^{a}\left(\boldsymbol{D}_{1} ; 2\right)$ (resp. $\left.\boldsymbol{e}^{a}\left(\boldsymbol{D}_{n} ; 2\right)\right)$ denotes the impossibility of recovering exactly $\boldsymbol{i}_{1}$ (resp. $\boldsymbol{i}_{n}$ ), even if $\boldsymbol{D}_{1}$ (resp. $\boldsymbol{D}_{n}$ ) had been perfectly available (a graphical description is given in Fig. (15)); this proves $(i)$.

\footnotetext{
${ }^{12}$ Note that even if the informative contribution of $\boldsymbol{D}_{i}$ is always $\lambda_{1, i}=1$ along the cross-range direction, its contribution in the FIM is weighted by $\frac{1}{r_{i}^{2}}$.
} 


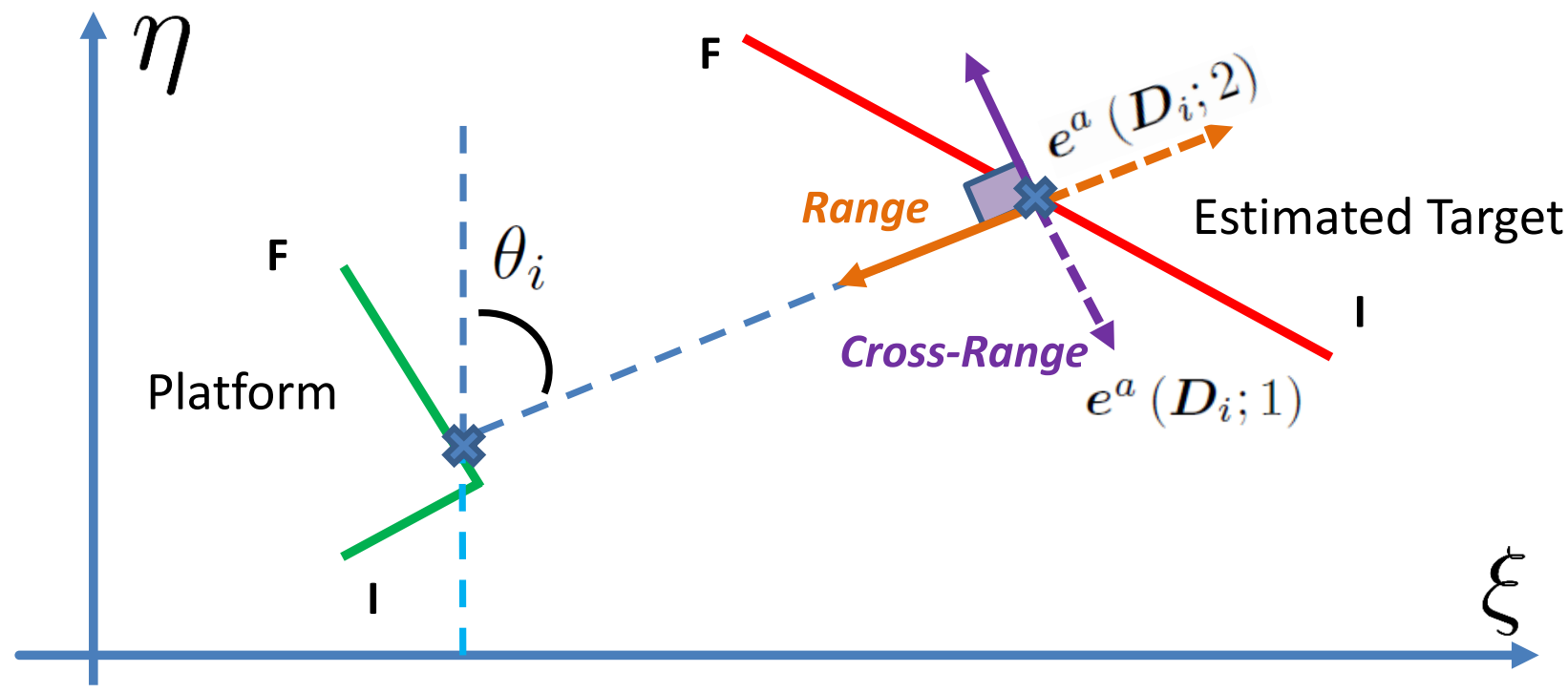

Figure 15: Eigenvectors ambiguity in the choice of $\left\{\hat{\boldsymbol{i}}_{1}, \hat{\boldsymbol{i}}_{n}\right\}$.

Before proceeding in the proof, it is worth noting that $\boldsymbol{e}^{a}\left(b_{i} \boldsymbol{D}_{i} ; t\right)=\boldsymbol{e}^{a}\left(\boldsymbol{D}_{i} ; t\right), t \in\{1,2\}$, $b_{i} \in \mathbb{R}^{+}$; for such a reason in the following w.l.o.g. we will search for a matrix $\boldsymbol{K}_{i}$ which approximates well $\boldsymbol{D}_{i}$, except for a scale factor $b_{i}$, i.e. $\boldsymbol{K}_{i} \approx b_{i} \boldsymbol{D}_{i}$; once $\boldsymbol{K}_{i}$ is obtained, the pairs $\left\{\hat{\boldsymbol{i}}_{1}^{p}, \hat{\boldsymbol{i}}_{n}^{q}\right\}, p, q \in\{-1,1\}$, are simply evaluated by considering the least informative eigenvectors of $\boldsymbol{K}_{1}$ and $\boldsymbol{K}_{n}$, respectively.

To obtain good estimates of $b_{1} \boldsymbol{D}_{1}$ and $b_{n} \boldsymbol{D}_{n}$ we can consider

$$
\begin{aligned}
& \boldsymbol{K}_{1} \triangleq \boldsymbol{J}^{o b s}[1,1]=\frac{\breve{\alpha}_{\theta}}{r_{1}^{2}} \boldsymbol{D}_{1}+\breve{\alpha}_{\theta} \sum_{i=2}^{n-1} \frac{\left(1-\alpha_{i}\right)^{2}}{r_{i}^{2}} \boldsymbol{D}_{i} \\
& \boldsymbol{K}_{n} \triangleq \boldsymbol{J}^{o b s}[2,2]=\frac{\breve{\alpha}_{\theta}}{r_{n}^{2}} \boldsymbol{D}_{n}+\breve{\alpha}_{\theta} \sum_{i=1}^{n-1} \frac{\alpha_{i}^{2}}{r_{i}^{2}} \boldsymbol{D}_{i}
\end{aligned}
$$

where in Eq. (83) (resp. Eq. (84)) we stress the (scaled) contribution of $\boldsymbol{D}_{1}$ (resp. $\boldsymbol{D}_{n}$ ) w.r.t. the spurious terms, i.e. $\boldsymbol{D}_{i}, i \in \mathcal{I} \backslash\{1\}$ (resp. $i \in \mathcal{I} \backslash\{n\}$ ). Exploiting again the inequalities among $\alpha_{i}$ and the assumptions of Eq. (81), it can be shown that $\frac{\breve{\alpha}_{\theta}}{r_{1}^{2}} \boldsymbol{D}_{1}$ (resp. $\frac{\breve{\alpha}_{\theta}}{r_{n}^{2}} \boldsymbol{D}_{n}$ ) is well approximated by Eq. (83) (resp. Eq. (84)); note that in this case convex combination counterparts are not needed because eigenvectors are not changed by a scaling factor. However, we will show hereinafter that a better estimate of $b_{i} \boldsymbol{D}_{i}$ can be obtained.

In fact let us consider $\boldsymbol{C}^{o b s} \triangleq\left(\boldsymbol{J}^{o b s}\right)^{-1}$ and denote $\boldsymbol{C}^{o b s}[\ell, m]$ as the $(\ell, m)$ th $[2 \times 2]$ block 
matrix of $C^{\text {obs }}$. By exploiting the block-wise inversion formula [5] we obtain

$$
\begin{aligned}
& \left(\boldsymbol{C}^{\text {obs }}[1,1]\right)^{-1}=\boldsymbol{J}^{o b s}[1,1]-\boldsymbol{J}^{o b s}[1,2] \boldsymbol{J}^{o b s}[2,2]^{-1} \boldsymbol{J}^{o b s}[1,2] \\
& \left(\boldsymbol{C}^{o b s}[2,2]\right)^{-1}=\boldsymbol{J}^{o b s}[2,2]-\boldsymbol{J}^{o b s}[1,2] \boldsymbol{J}^{o b s}[1,1]^{-1} \boldsymbol{J}^{o b s}[1,2]
\end{aligned}
$$

Exploiting the expression for $\boldsymbol{J}[\ell, m], \ell, m \in\{1,2\}$ as in Eqs. (76) and (77) and putting in evidence the scaled contribution of $\boldsymbol{D}_{1}$ (resp. $\boldsymbol{D}_{n}$ ), we get:

$$
\begin{aligned}
& \left(\boldsymbol{C}^{o b s}[1,1]\right)^{-1}=\frac{\breve{\alpha}_{\theta}}{r_{1}^{2}} \boldsymbol{D}_{1}+\breve{\alpha}_{\theta}\left\{\sum_{i=2}^{n-1} \frac{1}{r_{i}^{2}} \boldsymbol{D}_{i}\left[\left(1-\alpha_{i}\right)^{2} \boldsymbol{I}_{2}-\alpha_{i}\left(1-\alpha_{i}\right) \boldsymbol{T}_{1}\right]\right\} \\
& \left(\boldsymbol{C}^{o b s}[2,2]\right)^{-1}=\frac{\breve{\alpha}_{\theta}}{r_{n}^{2}} \boldsymbol{D}_{n}+\breve{\alpha}_{\theta}\left\{\sum_{i=2}^{n-1} \frac{1}{r_{i}^{2}} \boldsymbol{D}_{i}\left[\alpha_{i}^{2} \boldsymbol{I}_{2}-\alpha_{i}\left(1-\alpha_{i}\right) \boldsymbol{T}_{n}\right]\right\}
\end{aligned}
$$

where $\boldsymbol{T}_{1}$ and $\boldsymbol{T}_{n}$ are defined respectively as

$$
\begin{aligned}
& \boldsymbol{T}_{1} \triangleq\left[\left(\sum_{\ell=2}^{n} \frac{\alpha_{\ell}{ }^{2}}{r_{\ell}^{2}} \boldsymbol{D}_{\ell}\right)^{-1} \sum_{j=2}^{n-1} \frac{\alpha_{j}\left(1-\alpha_{j}\right)}{r_{j}^{2}} \boldsymbol{D}_{j}\right] \\
& \boldsymbol{T}_{n} \triangleq\left[\left(\sum_{\ell=1}^{n-1} \frac{\left(1-\alpha_{\ell}\right)^{2}}{r_{\ell}^{2}} \boldsymbol{D}_{\ell}\right)^{-1} \sum_{j=2}^{n-1} \frac{\alpha_{j}\left(1-\alpha_{j}\right)}{r_{j}^{2}} \boldsymbol{D}_{j}\right]
\end{aligned}
$$

It is apparent how each spurious term in the braces of Eqs. (87) and (88) (cf. with Eqs. (83) and (84)) is now filtered through the matrix gain $\boldsymbol{T}_{1}\left(\right.$ resp. $\left.\boldsymbol{T}_{n}\right)$ with weight $\alpha_{i}\left(1-\alpha_{i}\right)$. The matrix $\boldsymbol{T}_{1}$ (resp. $\boldsymbol{T}_{n}$ ) represents a "smoothing" factor (independent of $t_{i}$, cf. with Eqs. (83) and (84)). The weights $\alpha_{i}\left(1-\alpha_{i}\right)$ are such that there is a higher correction w.r.t. $\boldsymbol{D}_{i}$ corresponding to the the middle of the observation interval, while a little correction at the beginning or the end of the observation interval. However, while in the first case the correction tends to reduce the spurious term, in the latter case there is an increase of the error given by the spurious term.

To gain intuition about the effect of matrix $\boldsymbol{T}_{1}$ (same considerations apply to $\boldsymbol{T}_{n}$ in Eq. (88)) on Eq. (87) let us consider the case $r_{i} \approx r, \forall i \in \mathcal{I}$. In this case we have that $\boldsymbol{T}_{1} \approx$ $\left(\sum_{\ell=2}^{n} \alpha_{\ell}^{2} \boldsymbol{D}_{\ell}\right)^{-1} \sum_{j=2}^{n-1} \alpha_{j}\left(1-\alpha_{j}\right) \boldsymbol{D}_{j}$ and the the sum of the spurious terms in Eq. (87) reduces to

$$
\breve{\alpha}_{\theta}\left\{\frac{1}{r^{2}} \sum_{i=2}^{n-1} \boldsymbol{D}_{i}\left[\left(1-\alpha_{i}\right)^{2} \boldsymbol{I}_{2}-\alpha_{i}\left(1-\alpha_{i}\right) \boldsymbol{T}_{1}\right]\right\}
$$

Thus the magnitude of $\boldsymbol{T}_{1}$ will depend on the ratio of the concentration of $t_{i}$ at the middle of the observation interval by the concentration of $t_{i}$ at the end of the observation interval; in fact a higher ratio will imply a lower distortion in the smoothing of residual terms. 
Remark: Note that Eqs. (87) and (88) cannot be exploited to obtain better estimates of $\hat{r}_{1}$ and $\hat{r}_{n}$ than the ones in Eq. (33), even if the spurious terms are smoothed in such a case. The reason is that a proper normalization factor to obtain a convex combination cannot be found, since it can be shown that such a value would be dependent on $\boldsymbol{D}_{i}, i \in \mathcal{I}$, which are clearly not available.

\section{APPENDIX G \\ ChOICE OF $\hat{\boldsymbol{p}}_{p}\left(t_{k}\right)$}

In order to obtain $\hat{\boldsymbol{p}}_{P}\left(t_{k}\right)$ we will first seek an approximation of $\boldsymbol{p}_{P}\left(t_{m}\right)$ (denoted as $\tilde{\boldsymbol{p}}_{P}\left(t_{m}\right)$ ), where $t_{m} \triangleq \arg \min _{t_{i} \in \mathcal{T}}\left\|t_{i}-\frac{t_{n}-t_{1}}{2}\right\|_{2}$, i.e. the nearest $t_{i}$ to the middle of the observation interval. Similarly as $\left\{\breve{\boldsymbol{p}}_{P}\left(t_{1}\right), \breve{\boldsymbol{p}}_{P}\left(t_{n}\right)\right\}$ (cf. Eq. (31)), we can express $\breve{\boldsymbol{p}}_{P}\left(t_{m}\right)$ as

$$
\breve{\boldsymbol{p}}_{P}\left(t_{m}\right)=\hat{\boldsymbol{p}}_{T}\left(t_{m}\right)+r_{m} \boldsymbol{i}_{m}
$$

The estimate of $r_{m}$, denoted as $\tilde{r}_{m}$, is obtained, in analogy to Eq. (33), as

$$
\begin{aligned}
\tilde{r}_{m} & \triangleq \sqrt{\frac{\breve{\alpha}_{\theta} \sum_{i=1}^{n} \alpha_{i}\left(1-\alpha_{i}\right)}{\operatorname{tr}\left(\boldsymbol{J}^{o b s}[1,2]\right)}} \\
\operatorname{tr}\left(\boldsymbol{J}^{o b s}[1,2]\right) & =\breve{\alpha}_{\theta}\left[\frac{\alpha_{m}\left(1-\alpha_{m}\right)}{r_{m}^{2}}+\sum_{i=1, i \neq m}^{n} \frac{\alpha_{i}\left(1-\alpha_{i}\right)}{r_{i}^{2}}\right]
\end{aligned}
$$

As opposed to the case of $\left\{\boldsymbol{i}_{1}, \boldsymbol{i}_{n}\right\}$, the estimate of $\boldsymbol{i}_{m}$, denoted as $\tilde{\boldsymbol{i}}_{m}$, is obtained exploiting $\boldsymbol{J}^{o b s}[1,2]$ as a rough estimate of $b_{m} \boldsymbol{D}_{m}$, similarly as in Eqs. (83) and (84):

$$
\begin{aligned}
\tilde{\boldsymbol{i}}_{m} & \triangleq \boldsymbol{e}^{q}\left(\boldsymbol{J}^{\text {obs }}[1,2] ; 2\right) \\
\boldsymbol{J}^{\text {obs }}[1,2] & =\breve{\alpha}_{\theta}\left[\frac{\alpha_{m}\left(1-\alpha_{m}\right)}{r_{m}^{2}} \boldsymbol{D}_{m}+\sum_{i=1, i \neq m}^{n} \frac{\alpha_{i}\left(1-\alpha_{i}\right)}{r_{i}^{2}} \boldsymbol{D}_{i}\right] \\
q & \ni \operatorname{sign}\left\langle\boldsymbol{e}^{j}\left(\boldsymbol{J}^{o b s}[1,2] ; 1\right), \boldsymbol{u}\right\rangle=\operatorname{sign}\left\langle\hat{\boldsymbol{i}}_{1}, \boldsymbol{u}\right\rangle, \quad j \in\{-1,1\}
\end{aligned}
$$

The last line accounts for the sign ambiguity, in analogy to Eq. (39). The explicit form of $\tilde{\boldsymbol{p}}_{P}\left(t_{m}\right)$ is obtained by replacing $\left\{r_{m}, \boldsymbol{i}_{m}\right\}$ with $\left\{\tilde{r}_{m}, \tilde{\boldsymbol{i}}_{m}\right\}$ in Eq. (92). Some important remarks, about $\tilde{\boldsymbol{p}}_{P}\left(t_{m}\right)$, are in the following:

- Eq. (94) represents a weighted combination of the inverse squared ranges, in analogy to Eq. (33). However it is apparent that the weight corresponding to $\frac{1}{r_{m}^{2}}$, that is $\breve{\alpha}_{\theta} \alpha_{m}\left(1-\alpha_{m}\right)$, is weaker in this case w.r.t. the weights of the spurious terms. This leads not only to a poorer 
estimate $\tilde{r}_{m}$, as opposed to $\hat{r}_{1}$ and $\hat{r}_{n}$, but also $\tilde{r}_{m}$ will be biased toward the line between $r_{0}$ and $r_{n}$, leading to an estimated low-observable platform trajectory (which is not desirable as an initial guess);

- In Eq. (96) $\boldsymbol{J}^{o b s}[1,2]$ is used as a $b_{m} \boldsymbol{D}_{m}$ estimate, since a better estimate cannot be found, differently from $\left\{\boldsymbol{D}_{1}, \boldsymbol{D}_{n}\right\}$. In fact it can be shown that $\left(\boldsymbol{C}^{\text {obs }}[1,2]\right)^{-1}$ does not represent a better estimate of $b_{m} \boldsymbol{D}_{m}$. Therefore $\boldsymbol{J}^{\text {obs }}[1,2]$ represents the only existing approximation of $b_{m} \boldsymbol{D}_{m}$.

The above considerations suggest that one select a reasonable estimate of $\breve{\boldsymbol{p}}_{P}\left(t_{k}\right)$ according to a different approach, as described in the following.

In fact, we assume that the two legs form a $\pm \frac{\pi}{2}$ angle, since the platform needs to perform a maneuver that guarantees a good degree of observability; therefore $\left\{\breve{\boldsymbol{p}}_{P}\left(t_{1}\right), \breve{\boldsymbol{p}}_{P}\left(t_{k}\right), \breve{\boldsymbol{p}}_{P}\left(t_{n}\right)\right\}$ will form a right triangle. Furthermore the sign ambiguity in the turn leads to the definition of two specular vectors, denoted as $\boldsymbol{\rho}_{\ell}\left(t_{k}\right), \ell \in\{-1,1\}$.

Since $t_{k}$ is assumed known and $s$ is constant during the two legs, it can be shown, after geometric considerations, that such vectors are given by:

$$
\begin{aligned}
\boldsymbol{\rho}_{\ell}\left(t_{k}\right) & \triangleq \hat{\boldsymbol{p}}_{P}\left(t_{1}\right)+\frac{\left(t_{k}-t_{1}\right)}{\left(t_{n}-t_{k}\right)}\left\|\hat{\boldsymbol{p}}_{P}\left(t_{n}\right)-\hat{\boldsymbol{p}}_{P}\left(t_{1}\right)\right\|_{2} \cos (\nu)\left[\begin{array}{c}
\sin \left(\psi_{\ell}\right) \\
\cos \left(\psi_{\ell}\right)
\end{array}\right] \\
\psi_{\ell} & \triangleq \arctan _{2}\left(\hat{\boldsymbol{p}}_{P}\left(t_{n}\right)-\hat{\boldsymbol{p}}_{P}\left(t_{1}\right)\right)+\ell \cdot\left(\frac{\pi}{2}-\nu\right) \\
\nu & \triangleq \arctan \left(\frac{t_{k}-t_{1}}{t_{n}-t_{k}}\right)
\end{aligned}
$$

where $\nu$ and $\frac{\left(t_{k}-t_{1}\right)}{\left(t_{n}-t_{k}\right)}\left\|\hat{\boldsymbol{p}}_{P}\left(t_{n}\right)-\hat{\boldsymbol{p}}_{P}\left(t_{1}\right)\right\|_{2} \cos (\nu)$ represent the angle whose vertex is $\hat{\boldsymbol{p}}_{P}\left(t_{n}\right)$ and the distance between $\hat{\boldsymbol{p}}_{P}\left(t_{1}\right)$ and $\boldsymbol{\rho}_{\ell}\left(t_{k}\right)$, respectively. Finally, the unit vector $\left[\sin \left(\psi_{\ell}\right) \cos \left(\psi_{\ell}\right)\right]^{t}$ represents the direction from $\hat{\boldsymbol{p}}_{P}\left(t_{1}\right)$ to $\boldsymbol{\rho}_{\ell}\left(t_{k}\right)$ and accounts for the sign ambiguity in the turn, through the angle $\psi_{\ell}$.

The ambiguity is resolved by exploiting the coarse information given by Eqs. (94) and (96):

$$
\begin{aligned}
\hat{\boldsymbol{p}}_{P}\left(t_{k}\right) & =\boldsymbol{\rho}_{q}\left(t_{m}\right) \\
q & =\arg \min _{\ell \in\{-1,1\}}\left\|\tilde{\boldsymbol{p}}_{P}\left(t_{m}\right)-\boldsymbol{\rho}_{\ell}\left(t_{m}\right)\right\|_{2}
\end{aligned}
$$

where $\boldsymbol{\rho}_{\ell}\left(t_{m}\right)$ denotes the position vector at $t_{m}$ of the two-leg trajectory described by $\left\{\hat{\boldsymbol{p}}_{P}\left(t_{1}\right), \boldsymbol{\rho}_{\ell}\left(t_{k}\right), \hat{\boldsymbol{p}}_{P}\left(t_{n}\right)\right\}$. 


\section{REFERENCES}

[1] Y. Bar-Shalom, T. Kirubarajan, and X. R. Li, Estimation with Applications to Tracking and Navigation. New York, NY, USA: John Wiley \& Sons, Inc., 2001.

[2] Y. Bar-Shalom, P. K. Willett, and X. Tian, Tracking and Data Fusion: A Handbook of Algorithms. YBS publishing, 2011.

[3] F. Bavencoff, J. M. Vanpeperstraete, and J.-P. Le Cadre, "Constrained bearings-only target motion analysis via Markov chain Monte Carlo methods," IEEE Trans. Aerosp. Electron. Syst., vol. 42, no. 4, pp. 1240-1263, Oct. 2006.

[4] K. Becker, "Simple linear theory approach to TMA observability," IEEE Trans. Aerosp. Electron. Syst., vol. 29, no. 2, pp. 575-578, Apr. 1993.

[5] D. S. Bernstein, Matrix Mathematics: Theory, Facts, and Formulas with Application to Linear Systems Theory. Princeton University Press, Feb. 2005.

[6] W. R. Blanding, P. K. Willett, Y. Bar-Shalom, and R. Lynch, "Directed subspace search ML-PDA with application to active sonar tracking," IEEE Trans. Aerosp. Electron. Syst., vol. 44, no. 1, pp. 201-216, Jan. 2008.

[7] M. R. Chummun, Y. Bar-Shalom, and T. Kirubarajan, "Adaptive early-detection ML-PDA estimator for LO targets with EO sensors," IEEE Trans. Aerosp. Electron. Syst., vol. 38, no. 2, pp. 694-707, Apr. 2002.

[8] J. Clavard, D. Pillon, A.-C. Pignol, and C. Jauffret, "Bearings-only target motion analysis of a source in a circular constant speed motion from a non-maneuvering platform," in Proceedings of the 14th International Conference on Information Fusion (FUSION), Jul. 2011, pp. 1-8.

[9] K. Doğançay, “On the bias of linear least squares algorithms for passive target localization,” Signal Processing, vol. 84, no. 3, pp. 475-486, Mar. 2004.

[10] —_ "Passive emitter localization using weighted instrumental variables," Signal Processing, vol. 84, no. 3, pp. 487-497, 2004.

[11] _ _ "On the efficiency of a bearings-only instrumental variable estimator for target motion analysis," Signal Processing, vol. 85, no. 3, pp. 481-490, Mar. 2005.

[12] E. Fogel and M. Gavis, "Nth-order dynamics target observability from angle measurements," IEEE Trans. Aerosp. Electron. Syst., vol. 24, no. 3, pp. 305-308, May 1988.

[13] S. E. Hammel and V. J. Aidala, "Observability requirements for three-dimensional tracking via angle measurements," IEEE Trans. Aerosp. Electron. Syst., vol. AES-21, no. 2, pp. 200-207, Mar. 1985.

[14] J.-P. Le Cadre and C. Jauffret, "Discrete-time observability and estimability analysis for bearings-only target motion analysis," IEEE Trans. Aerosp. Electron. Syst., vol. 33, no. 1, pp. 178-201, Jan. 1997.

[15] _ _ "On the convergence of iterative methods for bearings-only tracking," IEEE Trans. Aerosp. Electron. Syst., vol. 35, no. 3, pp. 801-818, Jul. 1999.

[16] C. Jauffret, "Observability and Fisher information matrix in nonlinear regression," IEEE Trans. Aerosp. Electron. Syst., vol. 43, no. 2, pp. 756-759, Apr. 2007.

[17] C. Jauffret and Y. Bar-Shalom, “Track formation with bearing and frequency measurements in clutter," IEEE Trans. Aerosp. Electron. Syst., vol. 26, no. 6, pp. 999-1010, Nov. 1990.

[18] C. Jauffret and D. Pillon, "Observability in passive target motion analysis," IEEE Trans. Aerosp. Electron. Syst., vol. 32, no. 4, pp. 1290-1300, Oct. 1996.

[19] C. Jauffret, D. Pillon, and A.-C. Pignol, "Bearings-only maneuvering target motion analysis from a nonmaneuvering platform,” IEEE Trans. Aerosp. Electron. Syst., vol. 46, no. 4, pp. 1934-1949, Oct. 2010.

[20] S. M. Kay, Fundamentals of Statistical Signal Processing, Volume 1: Estimation Theory. Prentice Hall PTR, 1993. 
[21] T. Kirubarajan and Y. Bar-Shalom, "Low observable target motion analysis using amplitude information," IEEE Trans. Aerosp. Electron. Syst., vol. 32, no. 4, pp. 1367-1384, Oct.. 1996.

[22] J. C. Lagarias, J. A. Reeds, M. H. Wright, and P. E. Wright, "Convergence properties of the Nelder-Mead simplex method in low dimensions," SIAM Journal on Optimization, vol. 9, no. 1, pp. 112-147, 1998.

[23] S. C. Nardone and V. J. Aidala, “Observability criteria for bearings-only target motion analysis," IEEE Trans. Aerosp. Electron. Syst., vol. AES-17, no. 2, pp. 162-166, Mar. 1981.

[24] S. C. Nardone, A. Lindgren, and K. Gong, "Fundamental properties and performance of conventional bearings-only target motion analysis," IEEE Trans. Autom. Control, vol. 29, no. 9, pp. 775-787, Sep. 1984.

[25] J. M. Passerieux and D. V. Cappel, “Optimal observer maneuver for bearings-only tracking," IEEE Trans. Aerosp. Electron. Syst., vol. 34, no. 3, pp. 777-788, Jul. 1998.

[26] A. N. Payne, "Observability conditions for angles-only tracking," in Twenty-Second Asilomar Conference on Signals, Systems and Computers, vol. 1, 1988, pp. 451-457.

[27] S. Singer and S. Singer, "Complexity analysis of Nelder-Mead search iterations," in Proceedings of the first Conference on Applied Mathematics and Computation, 1999, pp. 185-196.

[28] Y. J. Zhang and G. Z. Xu, "Bearings-only target motion analysis via instrumental variable estimation," IEEE Trans. Signal Process., vol. 58, no. 11, pp. 5523-5533, Nov. 2010. 